\title{
Nonfeedback Distributed Beamforming Using Spatial-Temporal Extraction
}

\author{
Pongnarin Sriploy and Monthippa Uthansakul \\ School of Telecommunication Engineering, Suranaree University of Technology, Nakhon Ratchasima 30000, Thailand \\ Correspondence should be addressed to Monthippa Uthansakul; mtp@sut.ac.th
}

Received 29 October 2015; Revised 20 January 2016; Accepted 26 January 2016

Academic Editor: Lei Yu

Copyright (C) 2016 P. Sriploy and M. Uthansakul. This is an open access article distributed under the Creative Commons Attribution License, which permits unrestricted use, distribution, and reproduction in any medium, provided the original work is properly cited.

\begin{abstract}
So far, major phase synchronization techniques for distributed beamforming suffer from the problem related to the feedback procedure as a base station has to send the feedback reference signal back to the transmitting nodes. This requires stability of communication channel or a number of retransmissions, introducing a complicated system to both transmitter and receiver. Therefore, this paper proposes an alternative technique, so-called nonfeedback beamforming, employing an operation in both space and time domains. The proposed technique is to extract a combined signal at the base station. The concept of extraction is based on solving a simultaneous linear equation without the requirement of feedback or reference signals from base station. Also, the number of retransmissions is less compared with the ones available in literatures. As a result, the transmitting nodes are of low complexity and also low power consumption. The simulation and experimental results reveal that the proposed technique provides the optimum beamforming gain. Furthermore, it can reduce Bit Error Rate to the systems.
\end{abstract}

\section{Introduction}

Nowadays, wireless communication networks provide a variety of applications such as wireless local area networks, cellular networks, or wireless sensor networks. These wireless networks have lots of advantages in flexibility and mobility for users compared with a wired communication. However, the transmission range of wireless communication systems is limited due to signal attenuation [1]. Therefore, nodes or sensors in the systems require a higher transmitted power to compensate the mentioned attenuation. This requirement is not practical due to the limited battery lifetime of nodes. To tackle the problem, array antennas may be employed at individual devices in order to enhance beamforming gain [27]. Unfortunately, the installation of multiple antennas on a mobile terminal is difficult due to its size and the limitation of power consumption [8-10]. Recently, a distributed beamforming has been proposed to handle the mentioned problem $[11,12]$, which has lots of advantages such as a significant increase in transmission range and an enhancement of both energy efficiency and Signal-to-Noise Ratio (SNR) [13-16].

The distributed beamforming concept is similar to smart antennas but the position of antennas (or nodes) is not fixed.
Also, it could be said that the distributed beamforming is similar to virtual array antennas in which each node sends the same data at the same time to base station [17]. In the distributed beamforming networks, the transmitting nodes have to perform the synchronization in order to achieve phase alignment. Otherwise, the phase offsets or phase errors among all transmitted signals may degrade the combined signal at base station causing intersymbol distortion. Recently, lots of phase synchronization techniques have been proposed. These techniques can be classified into two types: closed-loop and open-loop synchronization techniques [18].

The closed-loop technique needs some feedback from base station to adjust the phase offsets among transmitting nodes. A one-bit feedback is one of the best techniques for closed-loop synchronization $[19,20]$. In the mentioned work, every transmitting node in the networks has to randomly adjust its carrier signal phases. Then, all nodes transmit the same data to base station. After performing an estimation of received SNR at base station, one bit (0 or 1) is transmitted back to all nodes. The bit " 0 " means that SNR is worse than before so that each node has to randomly adjust its phases again. On the other hand, bit "1" means that SNR is better 
than before so that all nodes have to update their latest phase adjustment. As we can see, this technique requires a large number of retransmissions from nodes to base station. For example, it requires at least $5 \mathrm{~N}$ iterations in order to achieve $75 \%$ guarantee of perfect or maximum beamforming gain, where $N$ stands for the number of transmitting nodes [21]. This may be considerably impractical as the battery life of nodes or mobile terminals is very limited. Moreover, a closedloop feedback from base station to transmitting nodes may be unreliable when the communication channel is weak.

In order to overcome the mentioned problems, two open-loop phase synchronization techniques, master-slave and time-slot round-trip techniques, have been proposed to reduce the interaction between base station and nodes. For master-slave technique, one node in the networks is selected as a master node while all remaining nodes are assigned to be slave nodes. The phase synchronization in this technique is achieved by sending the reference signals between master and slave nodes [21]. Alternatively, for time-slot round-trip technique, the phase synchronization among transmitting nodes can be obtained by sending a reference data among themselves $[22,23]$. The idea is based on the equivalence of round-trip transmission delays through a multihop chain between transmitting nodes and base station. According to these procedures, the open-loop techniques reduce the interaction among nodes and base station. However, both master-slave and round-trip techniques still require some feedback from base station. Also, this interaction increases a complexity to all transmitting nodes. In addition, nodes require a special hardware such as phase-locked loops to obtain a precise reference signal when performing phase synchronization.

Alternatively, a zero-feedback distributed beamforming technique has been lately proposed. This technique does not require any feedback signal from the base station and the unsynchronized carriers do not matter [24, 25]. However, this technique requires a large number of packet retransmissions. For example, in case of having 3 transmitting nodes, this technique requires at least 50 retransmissions (iterations) to obtain the beamforming gain at $9.1 \mathrm{~dB}$ [24]. Note that the maximum gain for this case is $9.5 \mathrm{~dB}$. In addition, the number of required retransmissions exponentially increases when the number of nodes increases.

From above literatures, the major disadvantage of existing phase synchronization techniques can be concluded as follows: the one-bit feedback and zero-feedback require a large number of retransmissions, which extremely reduces the battery life of transmitting nodes. Also, the masterslave and round-trip techniques require the reference signal among transmitting nodes which increases a complexity to transmitting nodes.

To overcome those disadvantages, a nonfeedback distributed beamforming technique is proposed in this paper. Note that the authors use "nonfeedback" term to avoid any confusion with the conceptual term defined for "zerofeedback" that appeared in $[24,25]$. In this paper, the proposed technique does not require any feedback signal from base station or interaction between transmitting nodes. Instead, the proposed technique requires a few number of retransmissions from nodes, which is only the same as the number of transmitting nodes, $N$. This is relatively small compared with the number of retransmissions for one-bit feedback and zero-feedback techniques. The proposed nonfeedback beamforming performs an extraction of combined signal at base station which means that the transmitting nodes do not need to deal with phase synchronization anymore; hence they can save energy and also battery life. The concept of extraction is based on a classical equation solving using inverse matrix. This procedure requires a few retransmissions from nodes. After performing a signal extraction, each extracted signal is properly weighted to obtain an appropriate phase alignment at base station. Finally, the base station obtains a combined signal with maximum beamforming gain. However, the retransmitted signals may be distorted when travelling through the communication channel.

This paper is organized as follows. Following an introduction including motivation and contribution of the proposed idea, the basic concept and definition of distributed beamforming techniques are presented in Section 2. Then, the proposed nonfeedback technique and its performance are discussed in Section 3. Afterwards, experimental studies are performed in Section 4 in order to validate the proposed concept. Finally, Section 5 concludes the paper.

\section{Distributed Beamforming: Concept and Definition}

The basic concept behind a distributed beamforming is similar to a traditional beamforming technique such as smart antennas in which the data is transmitted by array antennas located at one place. The phases of antennas are aligned so that the transmitted signals are gainfully combined at destination. For distributed beamforming networks, the transmitting nodes representing a group of single antenna elements are randomly distributed over the networks. As the nodes' locations are unknown the phase synchronization between nodes is the key challenge over a traditional beamforming. Figure 1 shows a configuration of distributed beamforming networks in which each transmitting node and base station are equipped with a single antenna element. All nodes and base station are stationary. It is assumed that $N$ distributed nodes transmit a shared message $x(t)$ to base station over the Rayleigh flat fading channel. The mathematical model of distributed beamforming networks is shown in Figure 2 which is detailed as follows. The received pass-band complex signal, $Y_{R}(t)$, can be written as

$$
Y_{R}(t)=\Re\left\{x(t) \sum_{n=1}^{N} h_{n} e^{j\left(\omega_{n} t+\phi_{n}\right)}+W(t)\right\},
$$

where $x(t)$ is a transmitted message, $h_{n}$ is a fading coefficient, and $A_{n}$ is a carrier signal amplitude at $n$th node. Also, $\omega_{n}=$ $\omega_{c}+\Delta \omega_{n}$, where $\omega_{c}$ is a carrier signal frequency and $\Delta \omega_{n}$ is a frequency offset. In addition, $\phi_{n}=\phi_{0}+\Delta \phi_{n}$, where $\phi_{0}$ is a nominal phase and $\Delta \phi_{n}$ is a phase offset of the signal coming from $n$th node which depends on the relative mobility or node location between $n$th node and base station. 


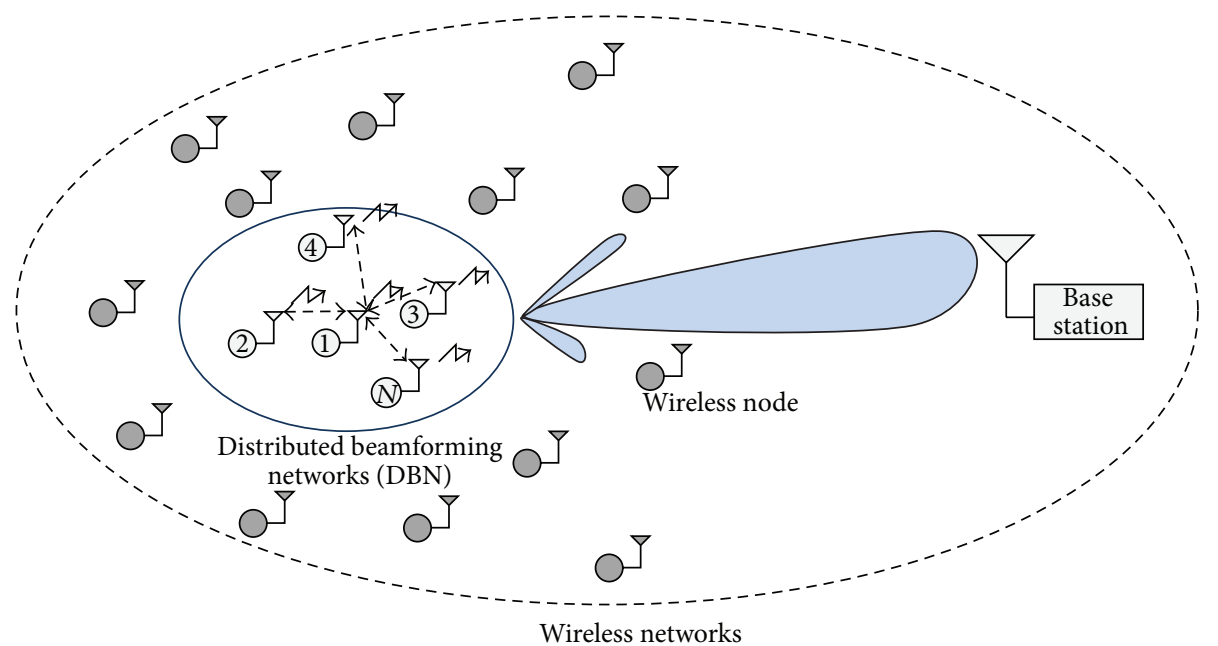

FIGURE 1: Configuration of wireless networks employing a distributed beamforming.

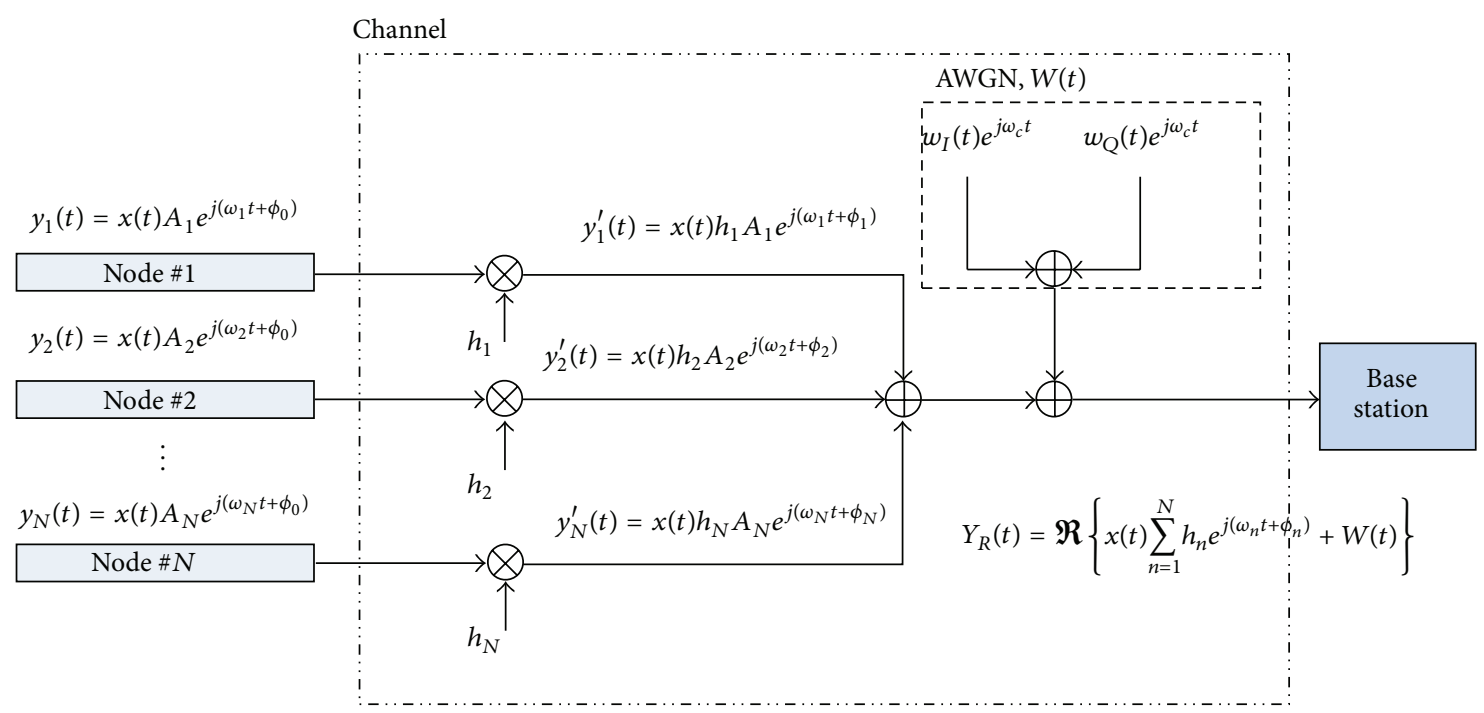

FIgURE 2: Mathematical model of wireless networks employing a distributed beamforming.

Furthermore, $W(t)$ is the Additive White Gaussian Noise (AWGN) which consists of in-phase, $w_{I}(t)$, and quadrature, $w_{Q}(t)$, components; that is, $W(t)=\left[w_{I}(t)+j w_{Q}(t)\right] e^{j \omega t}$. Therefore, the received signal power at base station can be expressed as follows [24]:

$$
\begin{aligned}
& \left|Y_{R}(t)\right|^{2}=x(t)^{2}\left\{\sum_{n=1}^{N} h_{n}^{2} A_{n}^{2}\right. \\
& \left.\quad+2 \sum_{n \neq m}\left[h_{n} h_{m}\right]\left[A_{n} A_{m}\right] \cos \left(\omega_{c} t-\omega_{c} t+\phi_{n}-\phi_{m}\right)\right\} \\
& +\left|W_{P B}(t)\right|^{2}=x(t)^{2}\left\{\sum_{n=1}^{N} h_{n}^{2} A_{n}^{2}\right. \\
& \left.\quad+2 \sum_{n \neq m}\left[h_{n} h_{m}\right]\left[A_{n} A_{m}\right] \cos \left(\phi_{n}-\phi_{m}\right)\right\}+\left|W_{P B}(t)\right|^{2} .
\end{aligned}
$$

The phase offset between each node $\left(\phi_{n}-\phi_{m}\right)$ shown in (2) is relatively significant to the beamforming gain. Note that $n$ and $m$ are the index of transmitting node in the networks in which $n \neq m$. In the case of having a finite number of nodes $N$, the distributed beamforming gain can be defined as a normalized received power at base station, $P_{R}$, as

$$
P_{R}=N\left\|\frac{x(t)}{N} \sum_{n=1}^{N}\left|h_{n}\right|^{2}\left|A_{n}\right|^{2} e^{j\left(\omega_{c} t+\phi_{n}\right)}\right\|^{2}+\|W(t)\|^{2} .
$$

As the phases of transmitting nodes are random, thus we consider the normalized received power in form of an expected value. If we assume that the received signal amplitude from all nodes is $A_{n}=1$, the average power of the transmitted signal is $P_{T}=1$, and $h_{n}$ are i.i.d. random variables then $E\left[h_{n}\right]=E[h]$ 
and $E\left[h_{n}^{2}\right]=1$. Therefore, the average power of the received signal is adopted from [21] as follows:

$$
\begin{aligned}
E & \left.P_{R}\right]=\frac{x(t)^{2}}{N} \\
\cdot & E\left[\sum_{n=1}^{N}\left|h_{n}\right|^{2}\left|A_{n}\right|^{2} e^{j\left(\omega_{c} t+\phi_{n}\right)} \sum_{m=1}^{N}\left|A_{m}\right|^{2} e^{j\left(\omega_{c} t+\phi_{m}\right)}\right] \\
& +E\left[\left|W_{P B}(t)\right|^{2}\right]=\frac{x(t)^{2}}{N}\left(N+\frac{N(N-1)}{2}\right. \\
& \left.\cdot E\left[\left|h_{n}\right|^{2}\left|A_{1}\right|^{2}\left|A_{2}\right|^{2} 2 \Re\left(e^{j\left(\omega_{c} t-\omega_{c} t+\phi_{1}-\phi_{2}\right)}\right)\right]\right) \\
& +E\left[\left|W_{P B}(t)\right|^{2}\right]=\frac{x(t)^{2}}{N}\left(N+\frac{N(N-1)}{2}\right. \\
& \left.\cdot 2 E\left[\cos \left(\phi_{1}-\phi_{2}\right)\right]\right)+E\left[\left|W_{P B}(t)\right|^{2}\right]=x(t)^{2}\{1 \\
& \left.+(N-1) E\left[\cos \left(\phi_{1}-\phi_{2}\right)\right]\right\}+E\left[\left|W_{P B}(t)\right|^{2}\right] \\
& =x(t)^{2}\{1+(N-1) \\
& \left.+E\left[\cos \left(\phi_{1}\right) \cos \left(\phi_{2}\right)-\sin \left(\phi_{1}\right) \sin \left(\phi_{2}\right)\right]\right\} \\
& +E\left[\left|W_{P B}(t)\right|^{2}\right]=x(t)^{2}\left\{1+(N-1) E\left[\cos \left(\phi_{i}\right)\right]^{2}\right\} \\
& +E\left[\left|W_{P B}(t)\right|^{2}\right],
\end{aligned}
$$

where $\phi_{i}$ is a phase offset among nodes which is uniformly distributed around $0^{\circ}$ between $[-\pi, \pi]$ interval. Figure 3 shows a normalized beamforming gain, $E\left[P_{R}\right]$, which is obtained using (4) upon varying the numbers of nodes in the networks. The "perfect distributed beamforming" term means that every received signal at base station is perfectly aligned. Note that the maximum beamforming gain is 1 as the signal normalization is performed at base station. For the case of having no phase offset ( or $\phi_{i}=0^{\circ}$ here defined as a perfect distributed beamforming), the resulting beamforming gain is of maximum value. Otherwise, the systems experience an unstable low beamforming gain. According to these results, phase synchronization is a key success for distributed beamforming which is the focus of this paper. As mentioned in the Introduction, various phase synchronization techniques have been proposed with a common drawback of sending a large number of feedback signals and the requirement of additional hardware. Alternatively, this paper proposes a phase synchronization technique avoiding any feedback signal from base station and any interaction between transmitting nodes. The proposed nonfeedback phase synchronization is described in the next section.

\section{Proposed Phase Synchronization}

In this section, we propose a nonfeedback distributed beamforming which does not require any interaction between nodes and feedback signals. The phase synchronization is performed at base station. At base station, there are two major parts: (1) RF front end operation which converts the RF

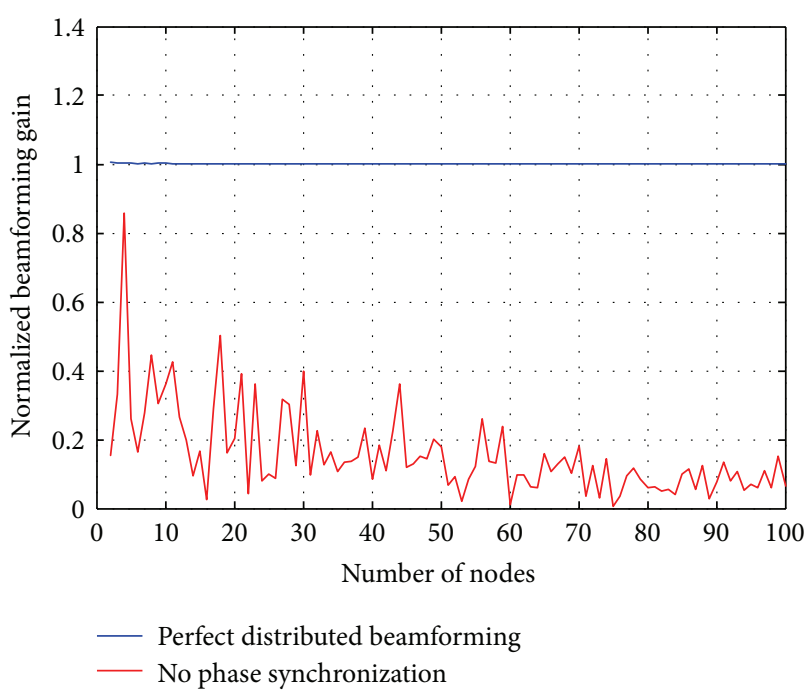

FIGURE 3: Beamforming gain in case of perfect beamforming and no phase synchronization.

signals to the baseband signals and (2) proposed nonfeedback technique procedure which performs signal extraction and phase synchronization. Moreover, the performance comparison between the proposed technique and some existing techniques is presented afterwards.

When base station receives signals from $N$ transmitting nodes, the composite received signal, $Y_{R}(t)$, is processed in the RF front end receiver in order to convert a pass-band received signal to digital baseband signal. The RF front end receiver consists of RF demodulator, band-pass filter, Analogto-Digital Converter (ADC), and Digital Downconverter (DDC). Then, the proposed nonfeedback technique is applied to the output baseband signal, $Y^{\prime \prime}(k)$, which includes a signal extraction and a weighting process. The mentioned procedures are detailed as follows.

3.1. RF Front End Operation. In RF front end, we assume that the received signal amplitude, $A_{n}$, is equal to 1 . The combined received signals at base station when they are coming from all transmitting nodes can be written as

$$
\begin{aligned}
Y_{R}(t)= & \Re\left\{\sum_{n=1}^{N} y_{n}^{\prime}(t)+W(t)\right\} \\
= & \Re\left\{x(t) \sum_{n=1}^{N} h_{n} e^{j\left(\omega_{n} t+\phi_{n}\right)}+W(t)\right\} \\
= & x(t) \sum_{n=1}^{N}\left[h_{n} \cos \left(\omega_{n} t+\phi_{n}\right)\right] \\
& +\underbrace{w_{I}(t)}_{\Re(t) \cos \left(\omega_{c} t\right)-w_{Q}(t) \sin \left(\omega_{c} t\right)},
\end{aligned}
$$

when $y_{n}^{\prime}(t)$ is a signal from the $n$th node, $\omega_{n}=\omega_{c}+\Delta \omega_{n}$ in which $\omega_{c}$ is a carrier signal frequency and $\Delta \omega_{n}$ is a frequency offset, $\phi_{n}=\phi_{0}+\Delta \phi_{n}$, where $\phi_{0}$ is a nominal phase, $\Delta \phi_{n}$ is a 


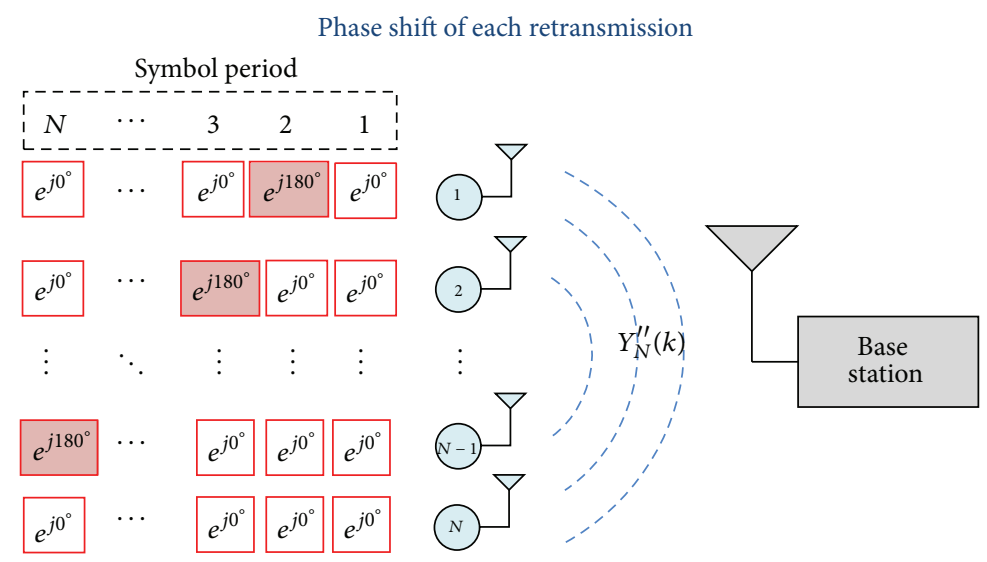

FIgURE 4: Proposed phase shifting pattern.

phase offset, and $W(t)$ is AWGN which consists of in-phase $w_{I}(t)$ and quadrature $w_{Q}(t)$ components. Then, the received signal that appeared in (5) is modulated and downconverted using RF modulator and DDC in order to obtain the digital baseband signal as follows:

$$
Y^{\prime \prime}(k)=x(k) \sum_{n=1}^{N} h_{n} e^{-j\left(\Delta \Omega_{n} k+\Phi_{n}\right)}+W_{B B}(k),
$$

where $k$ is a sampling time variable, $\Delta \Omega_{n}$ is a frequency offset, and $\Phi_{n}$ is a phase offset at $k$ th sampling time. In addition, $W_{B B}(k)$ is a baseband AWGN and $W_{B B}(k)=w_{I}(k)+j w_{Q}(k)$.

Equation (6) presents the baseband signal which can be degraded by the phase offset, $\Phi_{n}$. Thus, the baseband signal of each transmitting node requires a phase synchronization in order to obtain the maximum beamforming gain. Therefore, $Y^{\prime \prime}(k)$ is passed to the procedure of proposed nonfeedback technique which is presented in the following section.

3.2. Proposed Nonfeedback Technique. In this paper, a nonfeedback technique is proposed employing a signal extraction at base station to achieve a phase synchronization for distributed beamforming networks. The received signal, $Y^{\prime \prime}(k)$, mentioned earlier is processed in two steps: Spatial-Temporal Extraction and Optimum Weighting.

Step 1 (Spatial-Temporal Extraction). The combined signal that appeared in (6) needs to be extracted before performing a phase synchronization. The steps of signal extraction are as follows.

All transmitting nodes in the networks transmit the similar message to the base station at the same time. This transmission repeats only $N$ times where $N$ stands for the number of transmitting nodes in the networks. For each retransmission, the transmitting nodes adjust their phases according to a fixed phase adjustment pattern matrix, $\mathbf{A}_{N N}$, which can be obtained by the following algorithm. Note that this retransmission does not require any interaction among transmitting nodes:

(1) At 1st symbol period, all transmitting nodes send the signal without any phase adjustment.
(2) At the $n$th symbol period, where $n=[2,3, \ldots, N]$, only $(n-1)$ th transmitting node shifts its phase by $180^{\circ}$. For example, at the 2 nd symbol period, the 1 st node shifts its phase by $180^{\circ}$ and, at the 3rd symbol period, the 2 nd node shifts its phase by $180^{\circ}$ as seen in Figure 4 which shows the summary of proposed phase adjustment pattern.

(3) Repeat phase shifting pattern until $N$ symbol period.

According to the proposed phase adjustment pattern as presented above, we obtain the fixed coefficient matrix $\mathbf{A}_{N N}$ by retransmitting signal for $N$ times as shown in the following equations:

$$
\mathbf{A}_{N N}=\left[\begin{array}{ccccc}
1 & 1 & \cdots & 1 & 1 \\
e^{j 180^{\circ}} & 1 & \cdots & 1 & 1 \\
1 & e^{j 180^{\circ}} & \cdots & 1 & 1 \\
\vdots & \vdots & \ddots & \vdots & \vdots \\
1 & 1 & \cdots & e^{j 180^{\circ}} & 1
\end{array}\right] .
$$

After having completed $N$ retransmissions, all received signals are simultaneously arranged to form vector $\mathbf{Y}_{N}^{\prime \prime}$ as follows:

$$
\mathbf{Y}_{N}^{\prime \prime}(k)=\mathbf{A}_{N N} \mathbf{y}_{N}(k)+\mathbf{W}_{B B, N}(k)
$$

or

$$
\left[\begin{array}{c}
Y_{1}^{\prime \prime}(k) \\
Y_{2}^{\prime \prime}(k) \\
Y_{3}^{\prime \prime}(k) \\
\vdots \\
Y_{N}^{\prime \prime}(k)
\end{array}\right]=\underbrace{\left[\begin{array}{ccccc}
1 & 1 & \cdots & 1 & 1 \\
e^{j 180^{\circ}} & 1 & \cdots & 1 & 1 \\
1 & e^{j 180^{\circ}} & \cdots & 1 & 1 \\
\vdots & \vdots & \ddots & \vdots & \vdots \\
1 & 1 & \cdots & e^{j 180^{\circ}} & 1
\end{array}\right]}_{\mathbf{A}_{N N}}\left[\begin{array}{c}
y_{1}(k) \\
y_{2}(k) \\
y_{3}(k) \\
\vdots \\
y_{N}(k)
\end{array}\right]
$$




$$
+\left[\begin{array}{c}
W_{B B, 1}(k) \\
W_{B B, 2}(k) \\
W_{B B, 3}(k) \\
\vdots \\
W_{B B, N}(k)
\end{array}\right],
$$

where $\mathbf{Y}_{N}^{\prime \prime}(k)$ is the vector of combined received signal obtained by retransmitting signal for $N$ times. $\mathbf{y}_{N}(k)$ is the vector of transmitted message from $n$th node, $n=$ $[1,2, \ldots, N]$, and $\mathbf{W}_{B B, N}(k)$ is the vector of baseband AWGN. Equation (9) confirms that the retransmitting signal of $\mathbf{Y}_{N}^{\prime \prime}(k)$ provides the coefficient matrix $\mathbf{A}_{N N}$.

From the combined signal vector that appeared in (8), $\mathbf{Y}_{N}^{\prime \prime}(k)$ can be extracted by applying an inverse matrix $\mathbf{A}_{N N}^{-1}$ as seen in (10). Thus, we can extract the combined signal by utilizing the inverse matrix as follows:

$$
\mathbf{A}_{N N}^{-1} \mathbf{Y}_{N}^{\prime \prime}(k)=\mathbf{A}_{N N}^{-1} \mathbf{A}_{N N} \mathbf{y}_{N}(k)+\mathbf{A}_{N N}^{-1} \mathbf{W}_{B B, N}(k) .
$$

Then, the expression of $\mathbf{y}_{N}(k)$ that appeared in (8) becomes

$$
\mathbf{y}_{N}(k)+\mathbf{A}_{N N}^{-1} \mathbf{W}_{B B, N}(k)=\mathbf{A}_{N N}^{-1} \mathbf{Y}_{N}^{\prime \prime}(k) .
$$

Equation (11) represents the extracted signals from each node, $\mathbf{y}_{N}(k)+\mathbf{A}_{N N}^{-1} \mathbf{W}_{B B, N}(k)$ which can be extracted by applying the proposed $\mathbf{A}_{N N}^{-1}$. Equation (11) also reveals that the extracted signals are affected by baseband AWGN, $\mathbf{A}_{N N}^{-1} \mathbf{W}_{B B, N}(k)$.

This proposed technique will be demonstrated through an example of a beamforming network that is composed of four transmitting nodes, $N=4$. Note that each transmitting node and base station are equipped with a single antenna element. Also, all nodes are stationary and the operating frequency is $2.45 \mathrm{GHz}$. The received signals at base station are assumed to be equal to 1 having SNR of $20 \mathrm{~dB}$, referring to the minimum SNR of commercial Wi-Fi networks [26]. This confirms the feasibility of proposed concept when operated in real circumstances having rich noise signal. The phase offset is distributed over $-\pi$ to $\pi$. The utilized frequency offset is referred to as a typical frequency offset of clock crystals which is 1-20 parts per million (ppm) [24]. As the operating frequency is $2.45 \mathrm{GHz}$, the maximum frequency offset is $2.45 \mathrm{GHz} \times 20 \times 10^{-6}=49 \mathrm{kHz}$ and the minimum frequency offset is $2.45 \mathrm{GHz} \times 1 \times 10^{-6}=2.45 \mathrm{kHz}$. Thus, the frequency offset is distributed over $-49 \mathrm{kHz}$ to $49 \mathrm{kHz}$. As the systems are stationary, the effect of fading channel is now neglected. Thus, the received signal amplitude from all nodes is assumed to be 1 . As the focus of this paper is only phase synchronization, the perfect timing synchronization across all nodes is assumed in this system.

In case of $N=4$, expressions (8) can be rewritten as follows:

$$
\mathbf{Y}_{4}^{\prime \prime}(k)=\mathbf{A}_{44} \mathbf{y}_{4}(k)+\mathbf{W}_{B B, 4}(k)
$$

or

$$
\begin{aligned}
& {\left[\begin{array}{c}
Y_{1}^{\prime \prime}(k) \\
Y_{2}^{\prime \prime}(k) \\
Y_{3}^{\prime \prime}(k) \\
Y_{4}^{\prime \prime}(k)
\end{array}\right]=} \underbrace{\left[\begin{array}{cccc}
1 & 1 & 1 & 1 \\
e^{j 180^{\circ}} & 1 & 1 & 1 \\
1 & e^{j 180^{\circ}} & 1 & 1 \\
1 & 1 & e^{j 180^{\circ}} & 1
\end{array}\right]}_{\mathbf{A}_{44}}\left[\begin{array}{l}
y_{1}(k) \\
y_{2}(k) \\
y_{3}(k) \\
y_{4}(k)
\end{array}\right] \\
&+\left[\begin{array}{l}
W_{B B, 1}(k) \\
W_{B B, 2}(k) \\
W_{B B, 3}(k) \\
W_{B B, 4}(k)
\end{array}\right],
\end{aligned}
$$

where $Y_{1}^{\prime \prime}(k), Y_{2}^{\prime \prime}(k), Y_{3}^{\prime \prime}(k)$, and $Y_{4}^{\prime \prime}(k)$ are the received signal from retransmissions for the first, second, third, and fourth time, respectively. Also $y_{1}(k), y_{2}(k), y_{3}(k)$, and $y_{4}(k)$ are the transmitting signal, where $W_{B B, 1}(k), W_{B B, 2}(k), W_{B B, 3}(k)$, and $W_{B B, 4}(k)$ are baseband AWGN. Then, we can extract the original signal by utilizing the inverse matrix $\mathbf{A}_{44}^{-1}$ as follows:

$$
\mathbf{y}_{4}(k)+\mathbf{A}_{44}^{-1} \mathbf{W}_{B B, 4}(k)=\mathbf{A}_{44}^{-1} \mathbf{Y}_{4}^{\prime \prime}(k)
$$

or

$$
\begin{gathered}
{\left[\begin{array}{l}
y_{1}(k) \\
y_{2}(k) \\
y_{3}(k) \\
y_{4}(k)
\end{array}\right]+\underbrace{\left[\begin{array}{cccc}
0.5 & -0.5 & 0 & 0 \\
0.5 & 0 & -0.5 & 0 \\
0.5 & 0 & 0 & -0.5 \\
-0.5 & 0.5 & 0.5 & 0
\end{array}\right]}_{\mathbf{A}_{44}^{-1}}\left[\begin{array}{l}
W_{B B, 1}(k) \\
W_{B B, 2}(k) \\
W_{B B, 3}(k) \\
W_{B B, 4}(k)
\end{array}\right]} \\
=\underbrace{\left[\begin{array}{cccc}
0.5 & -0.5 & 0 & 0 \\
0.5 & 0 & -0.5 & 0 \\
0.5 & 0 & 0 & -0.5 \\
-0.5 & 0.5 & 0.5 & 0
\end{array}\right]\left[\begin{array}{c}
Y_{1}^{\prime \prime}(k) \\
Y_{2}^{\prime \prime}(k) \\
Y_{3}^{\prime \prime}(k) \\
Y_{4}^{\prime \prime}(k)
\end{array}\right] .}_{\mathbf{A}_{44}^{-1}}
\end{gathered}
$$

Figure 5(a) shows the original signals of 4 transmitting nodes which are separately sent to base station, $y_{1}(k), y_{2}(k)$, $y_{3}(k)$, and $y_{4}(k)$. As we can see in the figure, the initial phase of each signal is $4.5^{\circ}, 108.1^{\circ},-105.9^{\circ}$, and $-25.5^{\circ}$. Note that the phase of transmitting signals is random using uniform distribution. After all original signals are transmitted, they are destructively combined at base station. Figure 5(b) shows the 4 retransmissions of combined signals, $Y_{1}^{\prime \prime}(k), Y_{2}^{\prime \prime}(k)$, $Y_{3}^{\prime \prime}(k)$, and $Y_{4}^{\prime \prime}(k)$, for the first, second, third, and fourth time, respectively. As we can see, the maximum combination of received signal cannot be achieved as their phases are not suitably aligned. Thus, we propose an extraction of received signal at base station by applying the inverse matrix as shown in (14). After having done the proposed extraction, Figure 5(c) shows the 4 extracted signals $\mathbf{y}_{4}(k)+\mathbf{A}_{44}^{-1} \mathbf{W}_{B B, 4}(k)$ obtained from (14) or (15). As we can see in the comparison between Figures 5(a) and 5(c), phases of extracted signals are similar to original signals at $4.8^{\circ}, 110.7^{\circ},-104.3^{\circ}$, and 

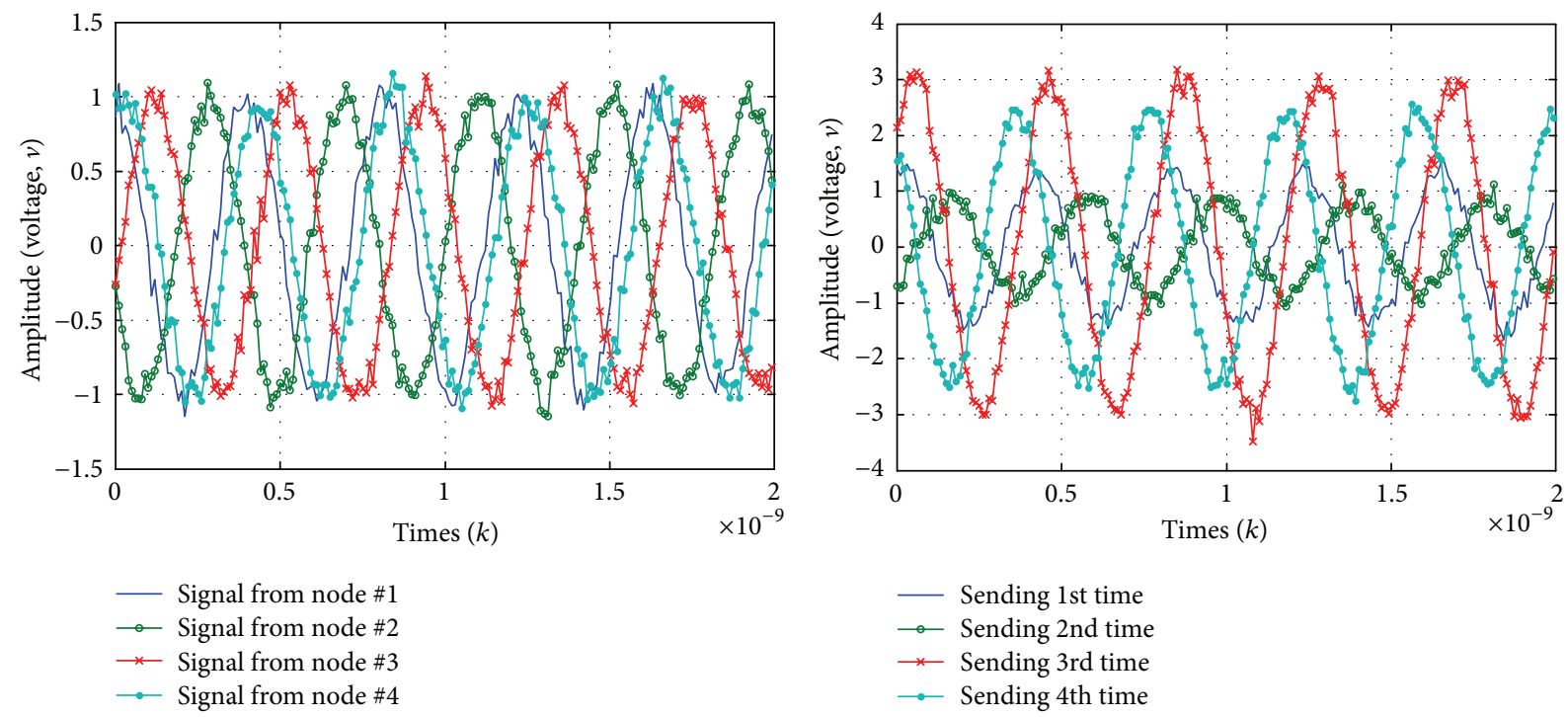

(a) Four original transmitted signals

(b) Combined retransmission signals

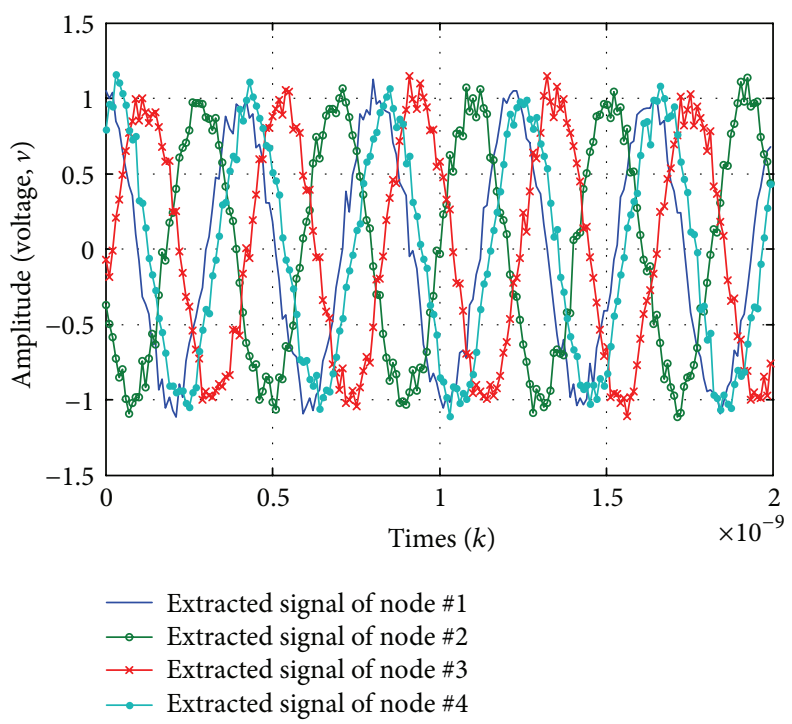

(c) Four extracted signals

Figure 5: Simulation results from Spatial-Temporal Extraction.

$-26.3^{\circ}$. At this point, phase offsets among those 4 signals still remain in which a suitable phase synchronization technique is required next.

Step 2 (Optimum Weighting). After we obtain the correct extracted signals as pointed out in previous step, the signals are sent to the weighting procedure. In this process, the phases of extracted signals will be synchronized by the following simple algorithm. The transmitted signal from the 1 st node is given to be a reference signal. Then, signals from remaining nodes $y_{n}(k)$ will be weighted by shifting their phases from $0^{\circ}$ to $360^{\circ}$ in order to find the best weighting coefficients which provide the maximum combined signal strength between the reference node $y_{1}(k)$ and the remaining nodes $y_{n}(k)$, where $n=[2,3, \ldots, N]$. As a result, the synchronized signals have equal phases referring to the chosen reference signal. Figure 6 presents the flow chart of the proposed phase synchronization concept. In the process of finding the best weighting coefficients, we can choose a weighting step size larger than $1^{\circ}$ in order to reduce the processing time. Figure 7 shows a normalized beamforming gain upon employing several weight steps varied from $1^{\circ}$ to $100^{\circ}$. In this simulation, we assume that the received signal amplitude from all nodes is equal to 1 having $\mathrm{SNR}=$ $20 \mathrm{~dB}$, the number of nodes is 20 , and the phase offset is uniformly distributed over $0^{\circ}$ to $360^{\circ}$. As we can see in this figure, a large weighting step provides the lower beamforming gain. This is because a large weighting step may skip the Optimum Weighting value. However, the weighting step of $\sim 10^{\circ}$ provides a similar beamforming gain as employing a 


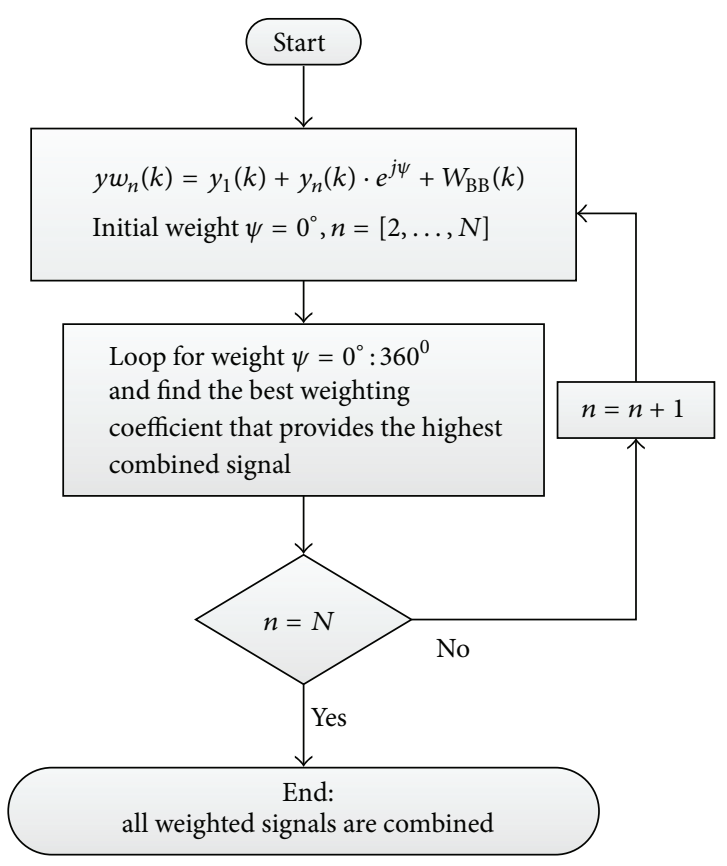

FIGURE 6: Summary flow chart of proposed phase synchronization.

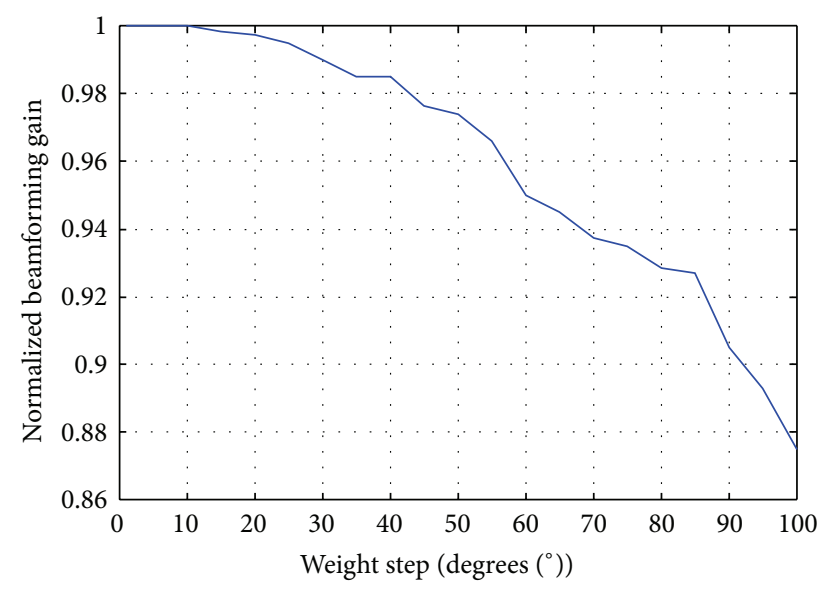

FIGURE 7: Normalized beamforming gain versus weighting step.

smaller weighting from $1^{\circ}$ to $10^{\circ}$. Thus, we can utilize the weighting step of $10^{\circ}$ to reduce the processing time.

After having done signal extraction and phase synchronization, we obtain the gainfully combined signal in a continuous time domain, $Y_{\mathrm{opt}}(t)$, as shown in Figure 8 . We finally obtain the maximum 4 times of transmitted signal amplitudes using the proposed techniques. The phase of combined signal equals the phase of the reference node $y_{1}(k)$. Without a phase synchronization, a lower beamforming gain is achieved due to the phase offset.

The simulation results in this section also reveal that the proposed nonfeedback technique has an efficiency over the one-bit feedback and zero-feedback techniques as the proposed technique requires a lower number of retransmissions comparing to the one-bit feedback and zero-feedback techniques. The proposed nonfeedback technique provides

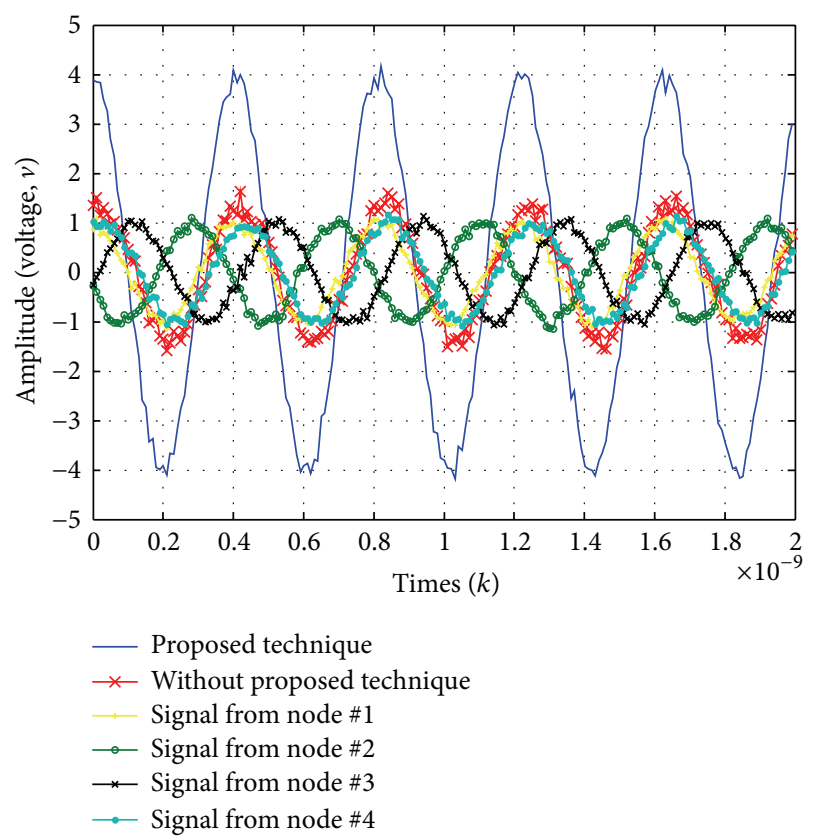

FIGURE 8: Received signal at base station from 4 transmitting nodes.

$N^{2} / N=N$ beamforming gain per transmission while the work presented in [18] has stated that the one-bit feedback offers $N^{2} / 5 N=N / 5$ beamforming gain per transmission with the requirement of at least $5 \mathrm{~N}$ retransmissions in order to achieve $75 \%$ guarantee of maximum beamforming gain. For example, considering employing 3 nodes in the networks, the proposed one and one-bit feedback offer gain per transmission of $3^{2} / 3=3$ and $3 / 5=0.6$, respectively. In addition, from [24], in case of having 3 transmitting nodes, the zerofeedback technique provides $3^{2} / 50=0.18$ beamforming gain per transmission as it requires at least 50 retransmissions in order to achieve $\approx 95 \%$ guarantee of maximum beamforming gain. As we can see, the proposed concept offers higher beamforming gain per one transmission comparing to other techniques.

The next section presents the beamforming gain comparison between the proposed technique and some existing phase synchronization techniques.

3.3. Performance Comparison. In this section, the average beamforming gains of some existing techniques and the proposed one are compared where the number of transmitting nodes is varied from 2 to 10 nodes. Note that the number of iterations for an average value is 100 . In the simulation, we assume that the received signals at base station have unit amplitudes, $A_{n}=1$, and SNR of $20 \mathrm{~dB}$. The random initial phase of each node is uniformly distributed over $-\pi$ to $\pi$. The effects of fading channel and Doppler are neglected. The number of retransmitting signals (retransmissions) is limited to 50 . Also, this paper focuses on only phase synchronization and the perfect timing and frequency synchronization across all transmitting nodes are assumed in this system. For the 


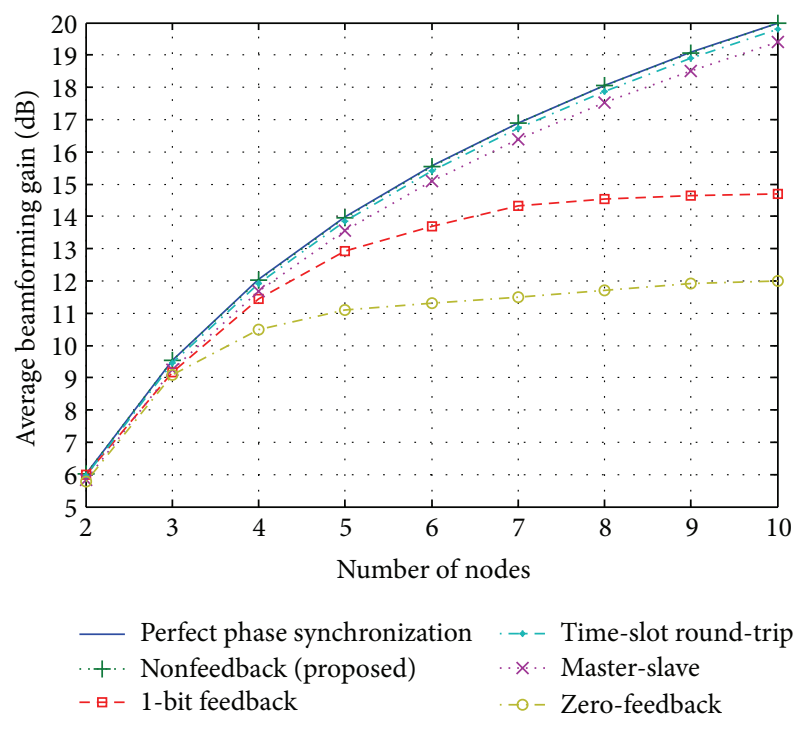

FIGURE 9: Beamforming gain of proposed nonfeedback technique versus other techniques.

proposed nonfeedback beamforming, we utilize the weighting step of $10^{\circ}$. Note that the reason of choosing the step size has been mentioned in the previous section.

As seen in Figure 9, the obtained results show that the average beamforming gains for the cases of proposed nonfeedback and time-slot round-trip are equal to the case of perfect phase synchronization. Although nonfeedback and time-slot round-trip techniques are comparable in terms of beamforming gain, the proposed nonfeedback technique is preferable as it does not require any feedback from base station while the time-slot round-trip technique requires a reference signal transmitted back from base station. Moreover, the proposed nonfeedback technique does not require any interaction between nodes while time-slot round-trip technique does. Although the master-slave technique also does not require any feedback from the base station it requires an interaction between transmitting nodes. This introduces a complexity to the systems. Moreover, the beamforming gain of the master-slave technique may be distorted by an uncompensated VCO phase drift. This phase drift occurred by the internal oscillator noise and over time of phase compensation in the open-loop mode, while the slave nodes are transmitting. Thus, the slave's carrier signals can be drifted out of phase [21].

According to the number of retransmissions which is limited to 50, the one-bit feedback and zero-feedback techniques provide lower beamforming gain compared with the proposed one. This is because the one-bit feedback and zerofeedback technique require a large number of retransmissions to achieve the maximum beamforming gain. The one-bit feedback technique requires the number of retransmissions to be at least $5 \mathrm{~N}$ retransmissions to achieve $75 \%$ guarantee of maximum beamforming gain [18]. Thus, the one-bit feedback requires the number of retransmissions to be larger than 50 to achieve the maximum beamforming gain upon having 10 transmitting nodes, $N=10$. Figure 9 shows that the 10 transmitting nodes for one-bit feedback technique provide $(14.7 \mathrm{~dB} / 20 \mathrm{~dB}) \times 100=73.5 \%$ of the maximum beamforming gain which is close to the numerical results of [18]. Figure 9 also presents that the one-bit feedback technique cannot provide the maximum beamforming gain when $N>3$. The reason is that only 50 retransmissions may be not enough to achieve the maximum beamforming gain while the zerofeedback requires at least 50 and 250 retransmissions to achieve $\approx 95 \%$ of the maximum beamforming gain upon having 3 and 4 transmitting nodes, respectively [24]. That means only 50 retransmissions are not enough to achieve the maximum beamforming gain when $N \geq 3$.

In summary, the proposed nonfeedback technique has the following advantages over other phase synchronization techniques: It offers higher effective gain with lower number of retransmissions. Also, it avoids interactions between transmitting nodes and also does not require any feedback signal from the base station.

\section{An Experimental Study of the Proposed Nonfeedback Distributed Beamforming Technique}

In a real circumstance, the proposed nonfeedback distributed beamforming can be affected by characteristic of communication channel such as phase variation or fading. Therefore, the experimental study of proposed techniques is considered in order to validate the proposed technique. A testbed consisting of two transmitting nodes and one base station was developed under SDR technology. We utilize a Universal Software Radio Peripheral (USRP) as it provides high speed ADCs, DACs, FPGA, and USB interface support [27, 28]. The experiments are separated into two parts: (A) an experiment for received signal power and (B) an experiment for BER. The first one is to prove if the proposed concept provides a gainfully combined signal at base station while the latter is to confirm the enhancement of system performance in terms of BER.

4.1. An Experiment on Received Signal Power. Figure 10 shows the configuration of experiment setup to measure the received signal power. A cosine wave is transmitted using USRP1 which includes two transmitting nodes, nodes \#A and $\#$ B. Note that XCVR2450 is employed as a daughter board for both cases. Then, base station receives the transmitted signal and conveys it to laptop for data recording. We use a single laptop in order to avoid the problem of timing synchronization among transmitting nodes. As the transmitted cosine wave is very sensitive to frequency offset, two daughter boards (or RF boards) on a single USPR1 are chosen. The USRP1 is connected to a laptop which is operated by Ubuntu 10.04. Figure 11(a) presents the configuration of transmitting nodes (\#A and $\# \mathrm{~B}$ ) which are placed at the sidewall. Figure 11(b) presents the placement of base station which is situated 7 meters away from the two transmitting nodes. According to a distance between the base station and laptop which is limited to about 2 meters (a maximum range of USB cable), we utilize a transmission line to extend a 


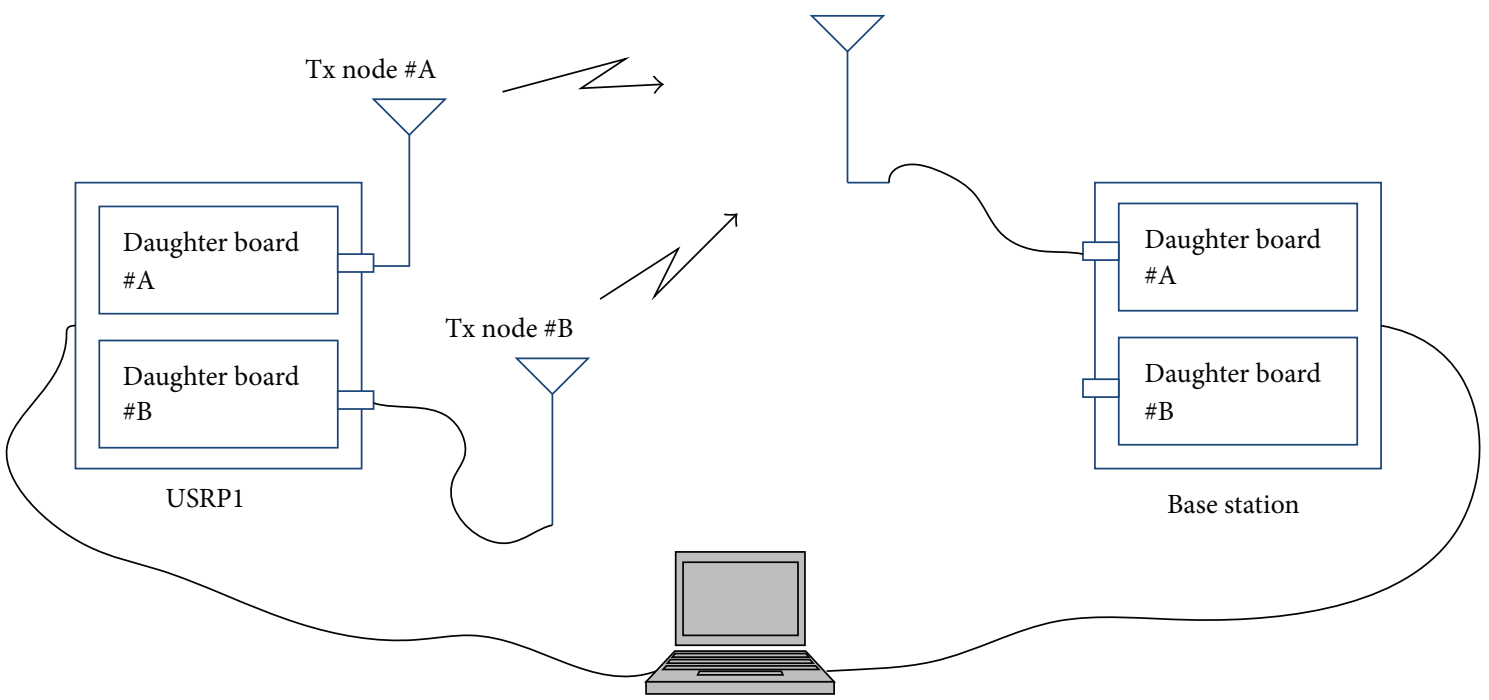

FIGURE 10: Configuration of measurement for received signal power.

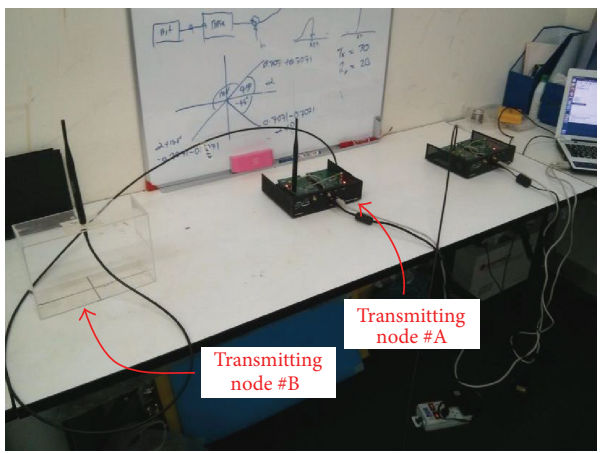

(a) Two transmitting nodes

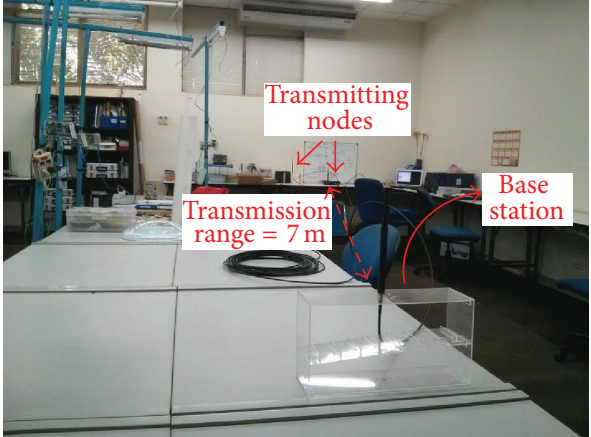

(b) Base station

FIGURE 11: The configuration of the experiment on received signal power.

communication range; the transmission lines are connected between USRP1 and antenna as shown in Figure 10. A loss of used transmission line is $-16.2 \mathrm{~dB}$. Therefore, the 7 meters is the longest distance to ensure that the received signal is not too weak. We cannot use a farther distance as the USRP is very sensitive for the signal strength. The antennas having gain of $3 \mathrm{dBi}$ are employed at both transmitting nodes and base station.

For the programming, we utilize GNU Radio Companion (GRC) version 3.7.4 which can build GNU Radio flow graphs using a graphical user interface. Figure 12(a) presents a block diagram of the transmitting nodes. Note that the phase differences between the two transmitting nodes are random by the node locations as shown in Figure 11(a). The "Signal Source" generates a cosine wave which has the setup parameters as follows: signal amplitude is 1 volt, signal frequency is $1 \mathrm{kHz}$, carrier frequency is $2.45 \mathrm{GHz}$, and sampling rate is $250 \mathrm{kHz}$. Then, the signal is weighted by a "Phase Shifter" considered as a weighting coefficient. This weighting coefficient depends on the proposed phase adjustment patterns shown in Figure 4.
Finally, the signals are transmitted by "UHD USRP Sink" where UHD is the USRP Hardware Driver compatible for all USRPs. Note that the transmitting gain of USRP parameter in GRC is $29 \mathrm{~dB}$. Note that the testbed has two transmitting nodes $(N=2)$. Thus, the required number of transmission is 2 times as the proposed nonfeedback beamforming technique requires only $N$ transmissions to perform beamforming when $N$ is the number of transmitting nodes. Figure 12(b) presents a block diagram of base station. The received signal is obtained by "UHD USRP Source." Then, the received signal is locked to the center frequency and downconverted to baseband signal by a "Costas Loop." The loop bandwidth of Costas Loop is 0.0065 radians per sample. Finally, the signal is saved at "File Sink." The saved files are used for an offline processing to be performed for the proposed technique as shown in Figure 12(c). The saved files are loaded by "File Source." The "File Source \#1" and "File Source \#2" are obtained at the 1st- and 2nd-time transmission, respectively. Then, the "Extraction" proposed in Section 3.2 provides the two extracted signals which related to the signal transmitted 
TABLE 1: Mean and standard deviation of measured average magnitude.

\begin{tabular}{lcccccc}
\hline & $1 \times 1 \mathrm{~A}$ & $1 \times 1 \mathrm{~B}$ & $2 \times 11$ st & $2 \times 12 \mathrm{nd}$ & $2 \times 1$ on & $2 \times 1$ off \\
\hline Mean (v) & 0.012 & 0.026 & 0.030 & 0.030 & 0.038 & 0.026 \\
Standard deviation (v) & 0.0022 & 0.0037 & 0.0042 & 0.0046 & 0.0051 & 0.0078 \\
\hline
\end{tabular}

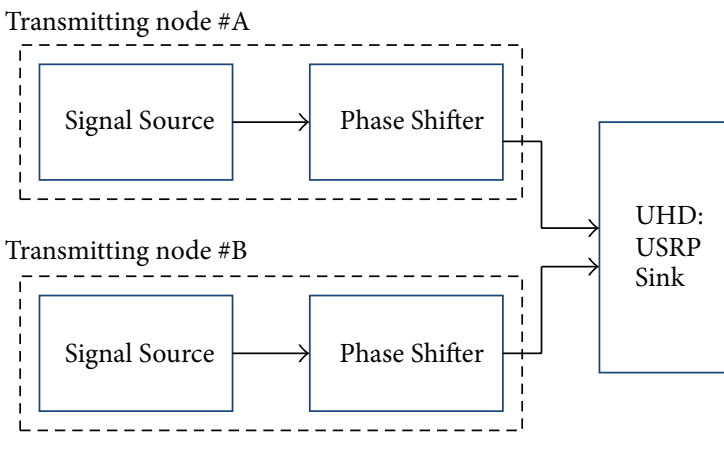

(a) Block diagram of transmitting nodes

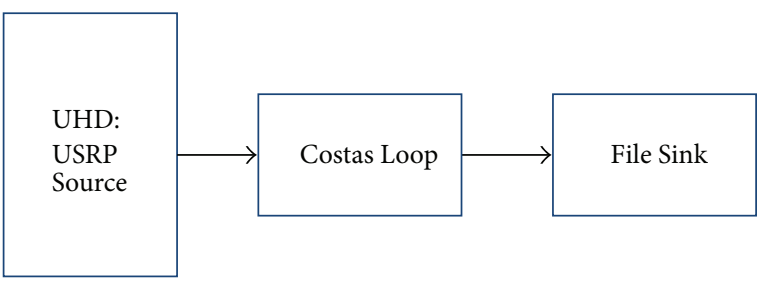

(b) Block diagram of base station

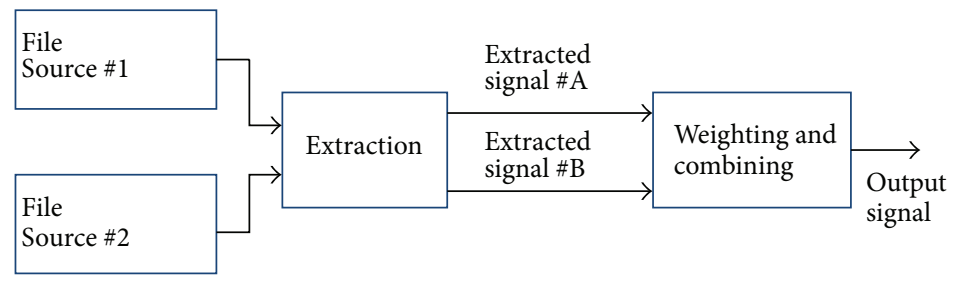

(c) Block diagram of the proposed technique

FIGURE 12: The programming block diagram for experiment on received signal power.

from nodes \#A and \#B. Finally, the extracted signals are weighted and combined according to proposed weighting algorithm discussed in Section 3.2.

The measured results are presented in a histogram of average combined magnitude at base station. Note that this magnitude is average from 100 -time data recording. Figure 13(a) shows the results in the case of only a single node (node \#A) that transmits a cosine wave to base station while Figure 13(b) is for the case when only node \#B transmits a cosine wave to base station. Figures 13(c) and 13(d) show the average of combined magnitude when both nodes \#A and \#B transmit a signal for the 1st time and 2nd time, respectively. Then, Figure 13(e) shows the output of combined signal when the proposed beamforming scheme has been performed. But when the proposed scheme is off (without phase synchronization), the combined signal turns to be lower as shown in Figure 13(f). As 100-time data of experiments is recorded, Table 1 shows a mean value and standard deviation of all cases. The results present that the proposed technique provides an optimum gain as 0.038 volts with respect to the optimum beamforming gain. Note that the optimum gain can be calculated by summation of the received signal power from nodes \#A and \#B $(0.012+0.026=0.038)$. Thus, the gain of proposed technique is significantly better than that without phase synchronization which provides a signal gain as only 0.026 volts. Moreover, a standard deviation in case of proposed technique ( $\sigma=0.0051$ ) is lower than that in the case when the proposed scheme is off $(\sigma=0.0078)$. This implies that the proposed technique provides higher stability in terms of received signal power.

The experimental results in this section validate that the proposed technique provides a gainfully combined signal at base station. However, the power of received signal cannot totally guarantee the quality of the received data. This is because the received signal can be affected by transmission channel such as fading, noise, interference, and bit synchronization between the two transmitting nodes. Therefore, we further investigate the BER in the next section.

4.2. An Experiment on Bit Error Rate. A testbed for BER measurement is shown in Figure 14. In this experiment, we transmit the random binary bits to a base station. The number of transmitting bits is 1 million which has a carrier frequency at $2.45 \mathrm{GHz}$. The USRP is employed at base station and two USRPB100s are employed as the transmitting nodes, nodes \#A and \#B. SBX-120 is used as the daughter boards for USRPB100. All USRPs are connected to a laptop for data recording. The two transmitting nodes are placed at the sidewall as shown in Figure 15. The configuration of the base station is the same as the one for previous experiment shown in Figure 11(b). Also, all losses in transmission line have been calibrated before performing the measurement. 


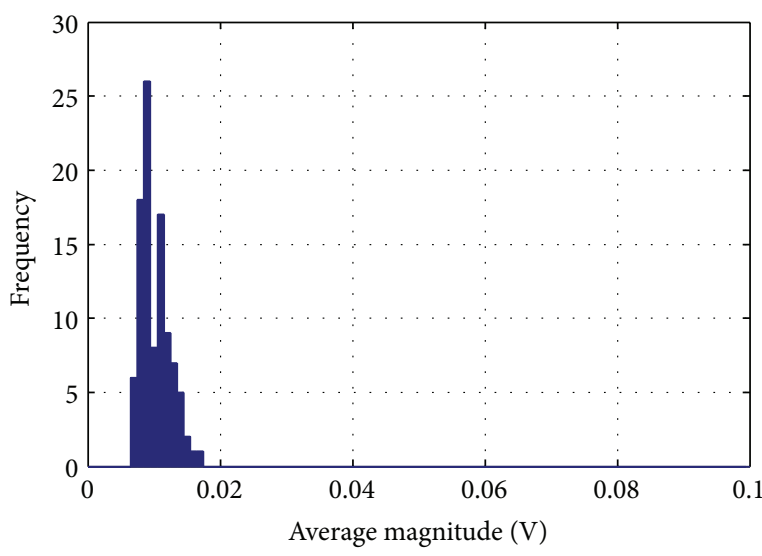

(a) Transmitting signal from node $\mathrm{A}(1 \times 1 \mathrm{~A})$

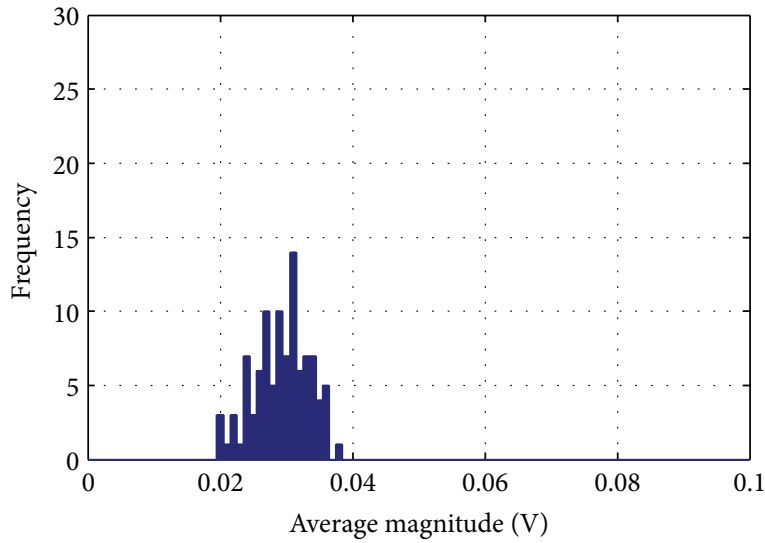

(c) Transmitting signal from two nodes at the 1st transmission $(2 \times 1$ 1st)

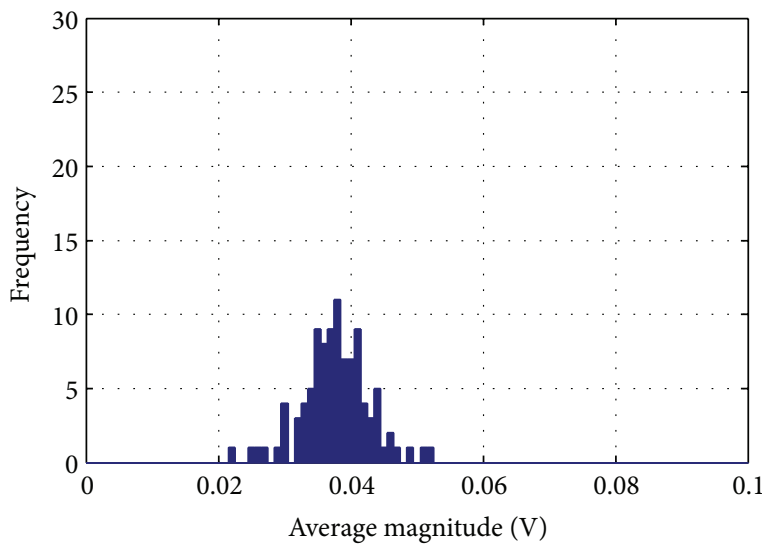

(e) Transmitting signal with proposed technique $(2 \times 1$ on $)$

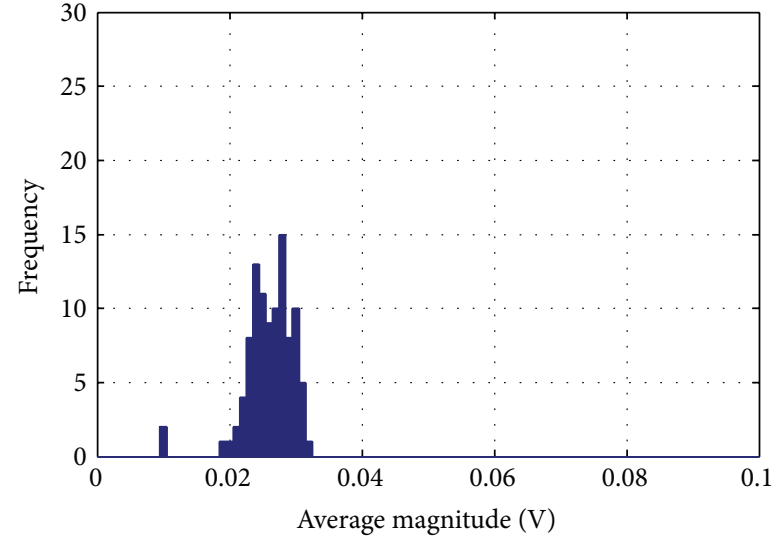

(b) Transmitting signal from node B $(1 \times 1 \mathrm{~B})$

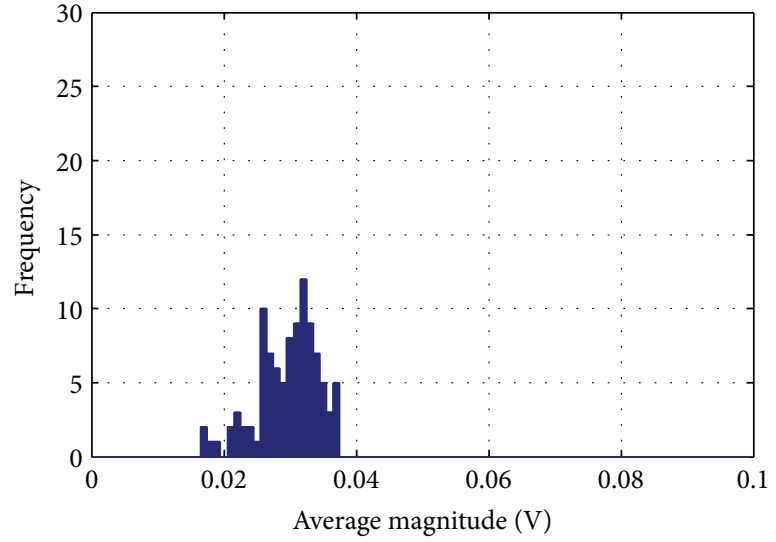

(d) Transmitting signal from two nodes at the 2 nd transmission $(2 \times$ 12 nd)

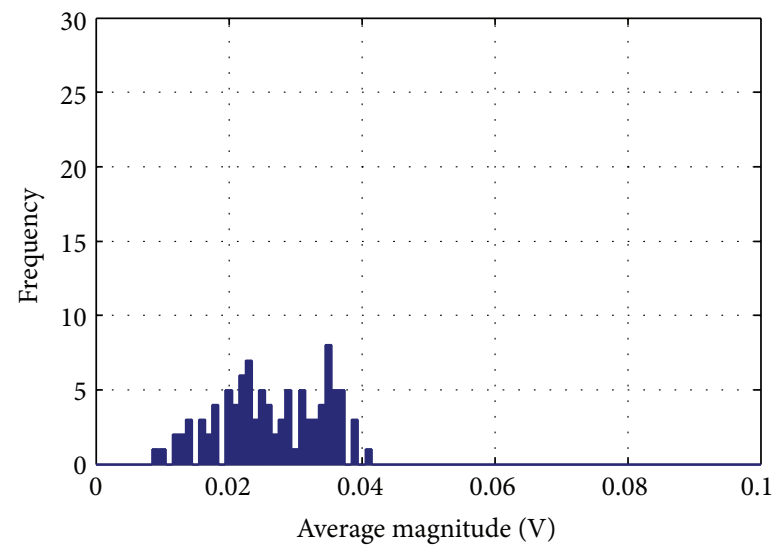

(f) Transmitting signal without phase synchronization $(2 \times 1$ off $)$

FIGURE 13: A histogram of average magnitude.

Figure 16(a) shows a block diagram of the transmitting nodes. The "Signal Source" generates the random binary bits which has sample rate of $250 \mathrm{kHz}$. Then, the signal is encoded by "Packet Encoder." In this block, the signal is wrapped into a packet which provides a payload length with a header, access code, and preamble. The setup parameters of this block are samples/symbol of 2 and bits/symbol of 1 . Afterwards, the encoded signals are demodulated by the "Differential
Binary Phase Shift Keying (DBPSK)" modulation. The setup parameter of this block is that an excess bandwidth (or roll-off factor) is 0.35 and Gray code is enabled. Then, the modulated signal is weighted by a "Phase Shifter" considered as weighting coefficient. Note that the mentioned weighting scheme has been proposed in Figure 4. Finally, the signals are transmitted at "UHD USRP Sink." The transmitted gain in GRC is $31 \mathrm{~dB}$ which related to the optimum transmitting gain. 


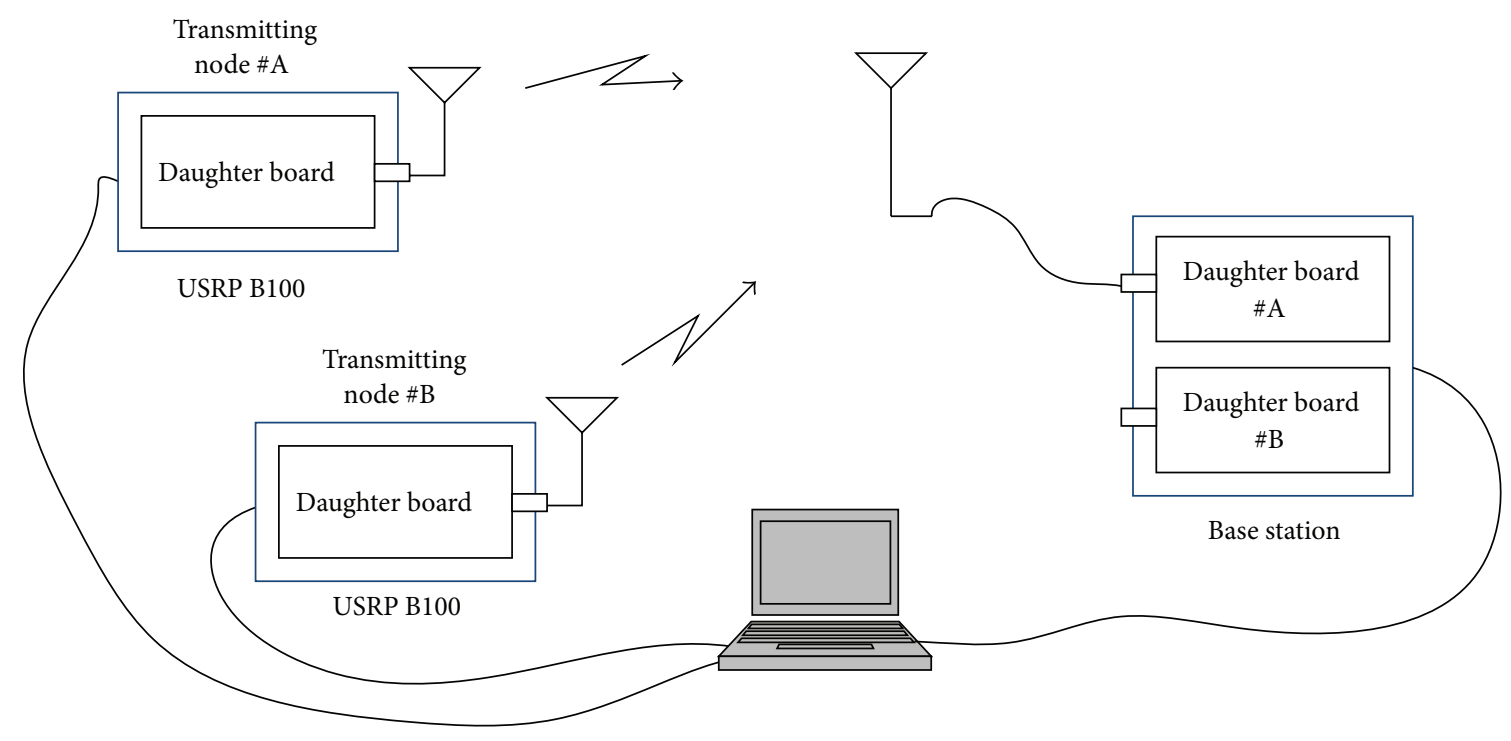

FIGURE 14: Configuration of measurement for BER.

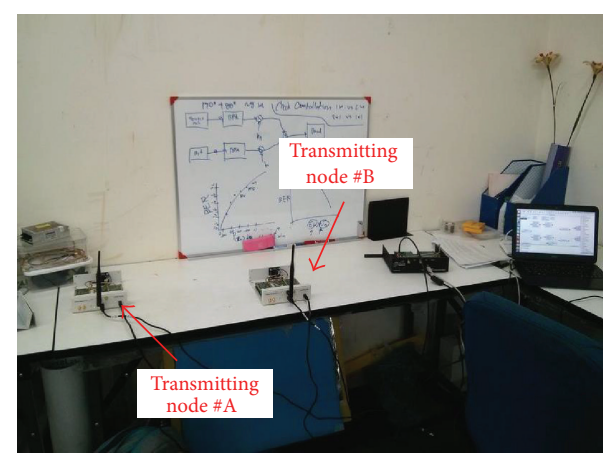

FIGURE 15: Two transmitting nodes employing USRP B100.

Figure 16(b) shows a block diagram of base station including "UHD USRP Sink" and "File Sink." In the offline processing, the saved files are extracted, weighted, and combined as shown in Figure 16(c). After that, the combined signals are demodulated using "DBPSK Demodulation." The setup parameters of this block are as follows: an excess bandwidth (or roll-off factor) is 0.35 , frequency lock loop bandwidth is 0.0628 radians per sample, phase recovery loop bandwidth is 0.0628 , timing recovery loop bandwidth is 0.0628 radians per sample, and Gray code is enabled. Finally, a demodulated signal is decoded by "Packet Decoder."

The measurement results are presented in a histogram of BER where 100-time recorded data has been averaged. Note that the measured BER employing USRP is relatively sensitive with noise. Thus, the major portion of measured BER is the optimum case as 0.0 or the worst case as 0.5 . Note that $\mathrm{BER}=0.0$ means that there is no bit error at all, while $\mathrm{BER}=0.5$ means that bit error turns out to be a half of transmitted bits; for example, bit error is 500,000 upon transmitting 1 million bits. Figure 17(a) presents the BER in the case of transmitting data from only node \#A while Figure 17(b) presents the case when only node \#B transmits the data. The results show that transmitting data from only a single node provides a low performance in terms of BER: the portion of $\mathrm{BER}=0.0$ which is only $11 / 100$ in the case of only node $\# \mathrm{~A}$ and the portion of $\mathrm{BER}=0.0$ which is only $22 / 100$ in the case of only node \#B. Then, the proposed technique is applied in order to enhance the BER. Figures 17(c) and 17(d) show the BER value at the base station when the two nodes transmit data at the 1st and 2nd time, respectively. Figure 17(e) shows the BER of combined signal at base station when the proposed technique has been applied. Figure 17(f) shows the BER for the case without the proposed technique. The results present that the proposed technique provides a lower BER than the case of transmitting signal from a single node and when the proposed technique is not applied. The portion of $\mathrm{BER}=0.0$ in case of using the proposed technique is $45 / 100$. The portion of BER $=0.0$ is only $25 / 100,17 / 100$, and 14/100 when the two nodes transmit data at the 1st and 2nd time and without phase synchronization, respectively. Table 2 shows a mean and standard deviation BER of all cases as the experiments have been recorded for 100 times. The results in this table confirm that the proposed technique makes the system BER lower comparing to other cases. The mean BER of the proposed technique is only 0.27 while the mean BER values in case of transmitting signal from a single node \#A and a single node \#B are 0.43 and 0.39 , respectively. The mean BER is $0.35,0.39$, and 0.40 when the two nodes transmit data at the 1st and 2 nd times and without phase synchronization, respectively. The BER performance obtained in the experiment is considered high as the received signals are too weak as shown in Table 1. However, the BER performance of proposed technique is significantly lower than the BER performance of using a single node $(1 \times 1 \mathrm{~A}$ and $1 \times 1 \mathrm{~B})$ and without phase synchronization $(2 \times 1$ off $)$. Therefore, the experimental results in this section validate 
TABLE 2: Mean and standard deviation of measured BER.

\begin{tabular}{lcccccc}
\hline & $1 \times 1 \mathrm{~A}$ & $1 \times 1 \mathrm{~B}$ & $2 \times 1$ st & $2 \times 1$ 2nd & $2 \times 1$ on & $2 \times 1$ off \\
\hline Mean (v) & 0.43 & 0.39 & 0.35 & 0.39 & 0.27 & 0.40 \\
Standard deviation (v) & 0.16 & 0.21 & 0.22 & 0.20 & 0.25 & 0.19 \\
\hline
\end{tabular}

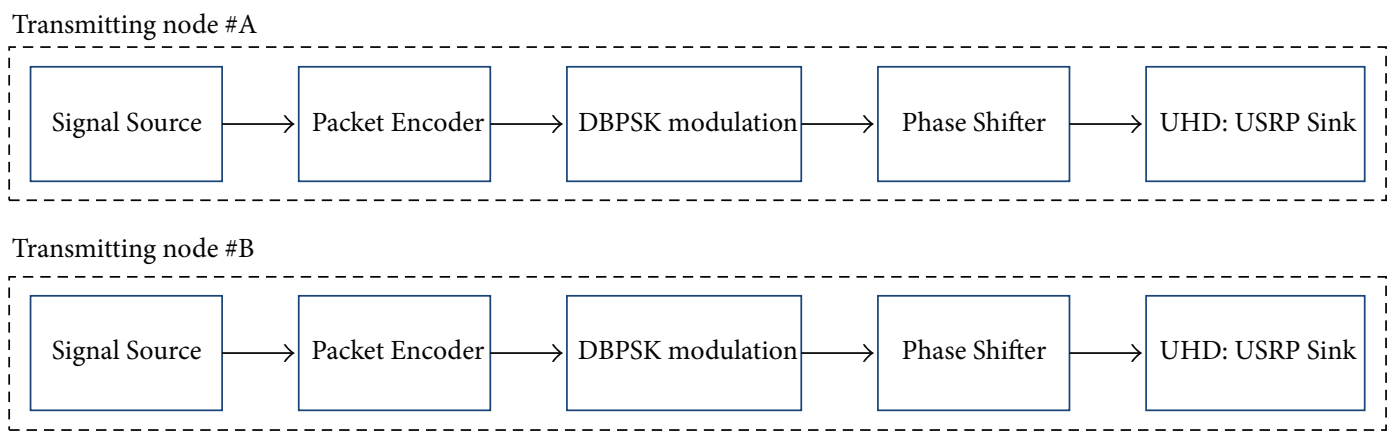

(a) A block diagram of the transmitting nodes

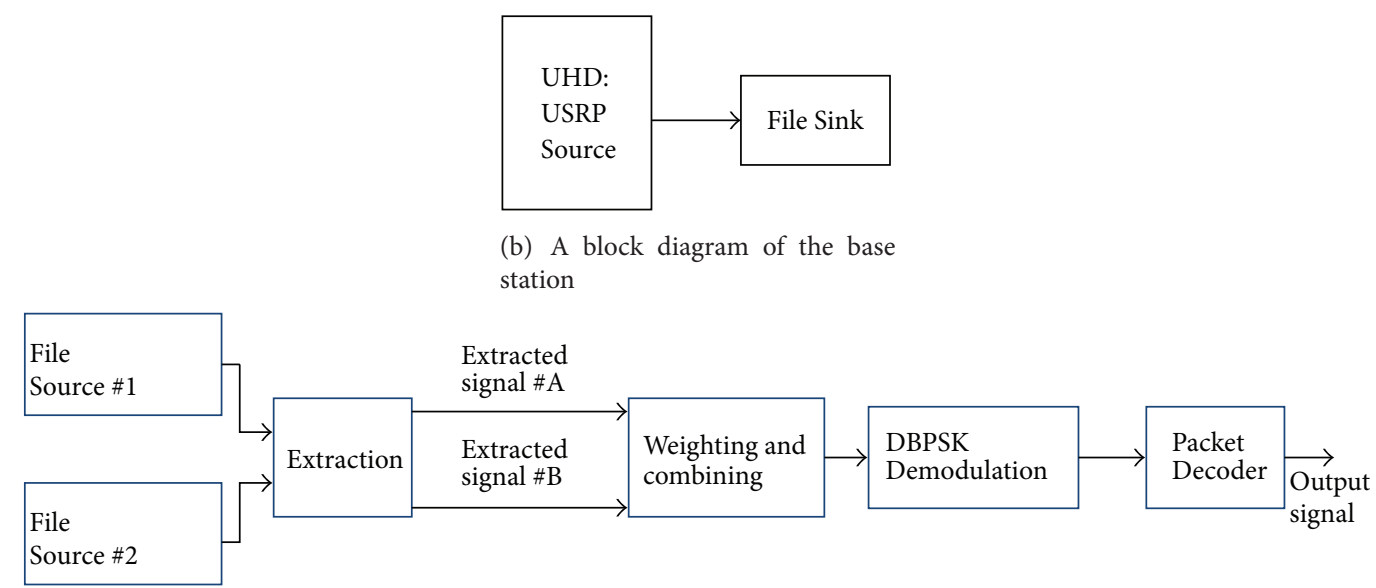

(c) A block diagram of the proposed technique

FIGURE 16: The programming block diagram experimental BER.

the proposed technique that it can be utilized to realize a distributed beamforming network with an optimum gain and lower BER.

\section{Conclusion}

This paper has proposed an alternative phase synchronization technique, so-called nonfeedback distributed beamforming technique. The proposed nonfeedback requires a lower number of retransmissions comparing to the one-bit feedback and zero-feedback techniques. Also, the proposed nonfeedback does not require any feedback signal or interaction between transmitting nodes. Using this technique, phase synchronization can be accomplished at base station instead of mobile terminals. From simulation results, the proposed nonfeedback technique provides a high beamforming gain compared with some existing phase synchronization techniques. Also, the proposed nonfeedback technique has been analyzed under real indoor environment. The measured results have revealed that the proposed technique provides the optimum beamforming gain. Also, it can enhance the system performance by lowering a Bit Error Rate (BER) comparing to the case when the phase synchronization is not applied.

\section{Conflict of Interests}

The authors declare that there is no conflict of interests regarding the publication of this paper. 


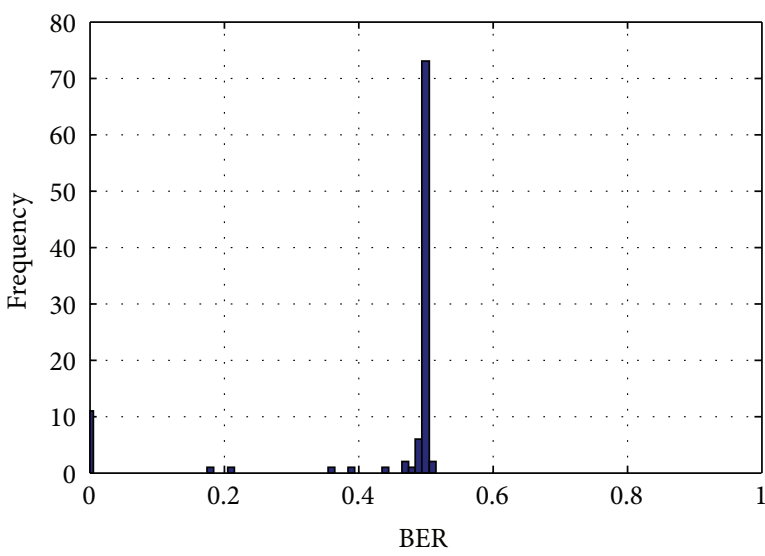

(a) Transmitting signal from node $\mathrm{A}(1 \times 1 \mathrm{~A})$

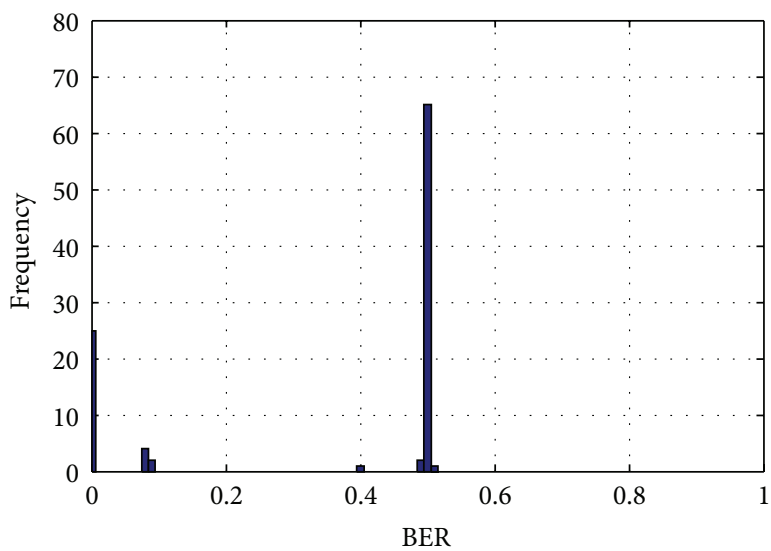

(c) Transmitting signal from two nodes at the 1st transmission $(2 \times 1$ 1st)

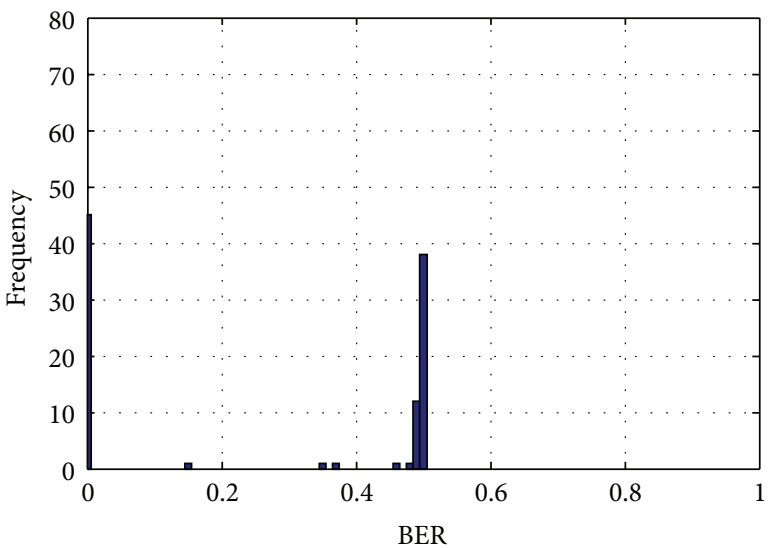

(e) Transmitting signal with proposed technique $(2 \times 1$ on $)$

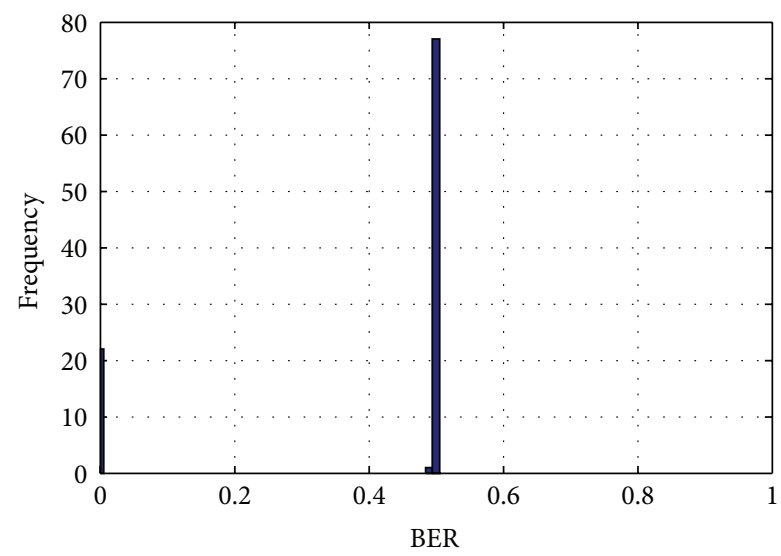

(b) Transmitting signal from node $\mathrm{B}(1 \times 1 \mathrm{~B})$

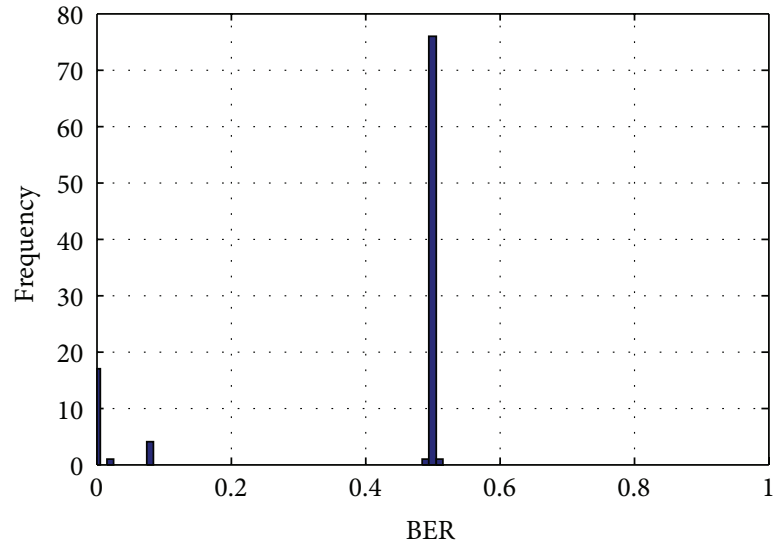

(d) Transmitting signal from two nodes at the 2 nd transmission $(2 \times$ 12 nd)

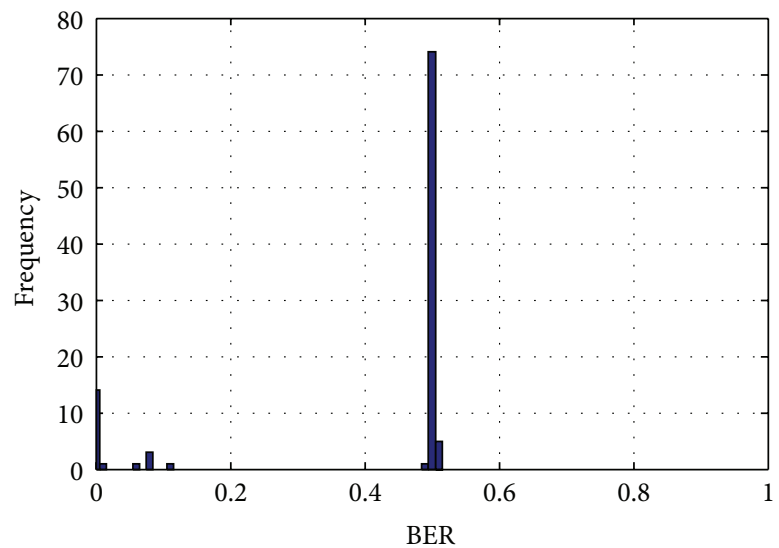

(f) Transmitting signal without phase synchronization $(2 \times 1$ off $)$

Figure 17: A histogram of Bit Error Rate.

\section{Acknowledgments}

This work was supported in part by The Royal Golden Jubilee Ph.D. (RGJ-Ph.D.) Program no. 1.Q.TS/52/B.1.B.XX and Suranaree University of Technology, Thailand.

\section{References}

[1] P. Sriploy, P. Uthansakul, and M. Uthansakul, "Effect of path loss on the distributed beamforming for Wireless Sensor Networks," in Proceedings of the 10th International Conference on Electrical Engineering/Electronics, Computer, Telecommunications and Information Technology (ECTI-CON '13), pp. 1-4, Krabi, Thailand, May 2013.

[2] C. A. Balanis, Antenna Theory: Analysis and Design, John Wiley \& Sons, New York, NY, USA, 2nd edition, 1997.

[3] P. Meerasri, P. Uthansakul, and M. Uthansakul, "Selfinterference cancellation-based mutual-coupling model for full-duplex single-channel MIMO systems," International 
Journal of Antennas and Propagation, vol. 2014, Article ID 405487, 10 pages, 2014.

[4] A. Innok, P. Uthansakul, and M. Uthansakul, "Angular beamforming technique for MIMO beamforming system," International Journal of Antennas and Propagation, vol. 2012, Article ID 638150, 10 pages, 2012.

[5] M. Uthansakul and M. E. Bialkowski, "Investigations into a wideband spatial beamformer employing a rectangular array of planar monopoles," IEEE Antennas and Propagation Magazine, vol. 47, no. 5, pp. 91-99, 2005.

[6] C. Bunsanit, P. Uthansakul, and M. Uthansakul, "Refinement method for weighting scheme of fully spatial beamformer," International Journal of Antennas and Propagation, vol. 2012, Article ID 359847, 13 pages, 2012.

[7] P. Uthansakul, A. Innok, and M. Uthansakul, "Open-loop beamforming technique for MIMO system and its practical realization," International Journal of Antennas and Propagation, vol. 2011, Article ID 723719, 13 pages, 2011.

[8] F. Rayal, "Why have smart antennas not yet gained traction with wireless network operators?" IEEE Antennas and Propagation Magazine, vol. 47, no. 6, pp. 124-126, 2005.

[9] T. Kaiser, "When will smart antennas be ready for the market? Part I," IEEE Signal Processing Magazine, vol. 22, no. 2, pp. 8792, 2005.

[10] M. Kosanovic and M. Stojcev, "Sensor node lifetime prolonging," in Proceedings of the 20th Telecommunications Forum (TELFOR '12), pp. 178-181, Belgrade, Serbia, November 2012.

[11] Y. T. Lo, "A mathematical theory of antenna arrays with randomly spaced elements," IEEE Transactions on Antennas and Propagation, vol. 12, no. 3, pp. 257-268, 1964.

[12] H. Ochiai, P. Mitran, H. V. Poor, and V. Tarokh, "Collaborative beamforming for distributed wireless ad hoc sensor networks," IEEE Transactions on Signal Processing, vol. 53, no. 11, pp. 41104124, 2005.

[13] P. Sriploy, P. Uthansakul, and M. Uthansakul, "The optimum number of nodes and radius for distributed beamforming networks," ECTI Transactions on Electrical Engineering, Electronics, and Communications (EEC), vol. 13, no. 2, pp. 35-47, 2014.

[14] S. Amini, D.-J. Na, and K. Choi, "Performance comparison between distributed beamforming and clustered beamforming," in Proceedings of the 11th International Conference on Information Technology: New Generations (ITNG '14), pp. 174-179, IEEE, Las Vegas, Nev, USA, April 2014.

[15] L. Yang, K. A. Qaraqe, E. Serpedin, and M.-S. Alouini, "Performance analysis of distributed beamforming in a spectrum sharing system," IEEE Transactions on Vehicular Technology, vol. 62, no. 4, pp. 1655-1666, 2013.

[16] M. Dohler and Y. Li, Cooperative Communications: Hardware, Channel and PHY, John Wiley \& Sons, Hoboken, NJ, USA, 2010.

[17] K. Yao, R. E. Hudson, C. W. Reed, D. Chen, and F. Lorenzelli, "Blind beamforming on a randomly distributed sensor array system," IEEE Journal on Selected Areas in Communications, vol. 16, no. 8, pp. 1555-1567, 1998.

[18] R. Mudumbai, D. R. Brown III, U. Madhow, and H. V. Poor, "Distributed transmit beamforming: challenges and recent progress," IEEE Communications Magazine, vol. 47, no. 2, pp. 102-110, 2009.

[19] R. Mudumbai, J. Hespanha, U. Madhow, and G. Barriac, "Scalable feedback control for distributed beamforming in sensor networks," in Proceedings of the IEEE International Symposium on Information Theory (ISIT '05), pp. 137-141, Adelaide, Australia, September 2005.

[20] R. Mudumbai, J. Hespanha, U. Madhow, and G. Barriac, "Distributed transmit beamforming using feedback control," IEEE Transactions on Information Theory, vol. 56, no. 1, pp. 411426, 2010.

[21] R. Mudumbai, G. Barriac, and U. Madhow, "On the feasibility of distributed beamforming in wireless networks," IEEE Transactions on Wireless Communications, vol. 6, no. 5, pp. 1754-1763, 2007.

[22] D. R. Brown and H. V. Poor, "Time-slotted round-trip carrier synchronization for distributed beamforming," IEEE Transactions on Signal Processing, vol. 56, no. 11, pp. 5630-5643, 2008.

[23] D. R. Brown III, B. Zhang, B. Svirchuk, and M. Ni, "An experimental study of acoustic distributed beamforming using round-trip carrier synchronization," in Proceedings of the 4th IEEE International Symposium on Phased Array Systems and Technology (Array '10), pp. 316-323, Waltham, Mass, USA, October 2010.

[24] A. Bletsas, A. Lippman, and J. N. Sahalos, "Simple zero-feedback distributed beamforming with unsynchronized carriers," IEEE Journal on Selected Areas in Communications, vol. 28, no. 7, pp. 1046-1054, 2010.

[25] G. Sklivanitis and A. Bletsas, "Testing zero-feedback distributed beamforming with a low-cost SDR testbed," in Proceedings of the 45th Asilomar Conference on Signals, Systems and Computers (ASILOMAR '11), pp. 104-108, Pacific Grove, Calif, USA, November 2011.

[26] Wi-Fi: Define Minimum SNR Values for Signal Coverage, May 2015, http://www.enterprisenetworkingplanet.com/netsp/ article.php/3747656/.

[27] C. Clark, Software Defined Radio: With GNU Radio and USRP, McGraw-Hill, New York, NY, USA, 2008.

[28] Ettus Research Company, April 2015, http://www.ettus.com/. 


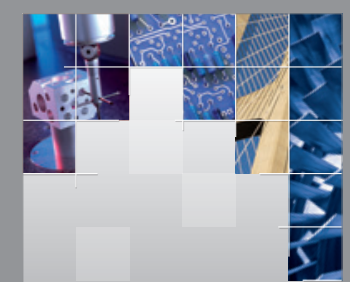

\section{Enfincering}
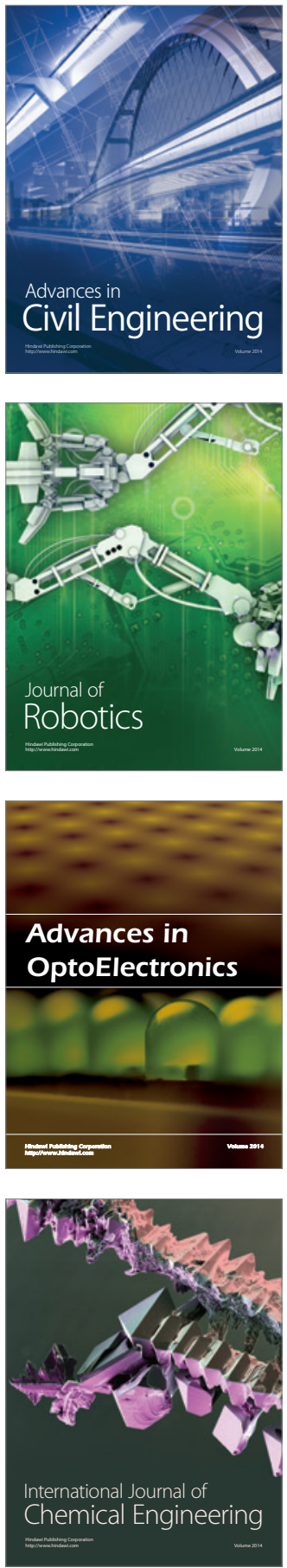

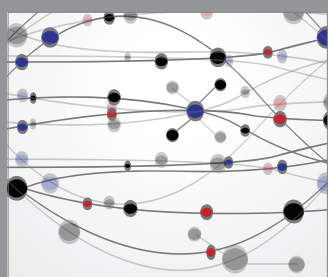

The Scientific World Journal

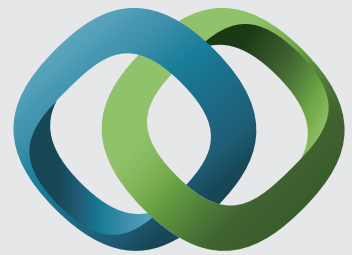

\section{Hindawi}

Submit your manuscripts at

http://www.hindawi.com
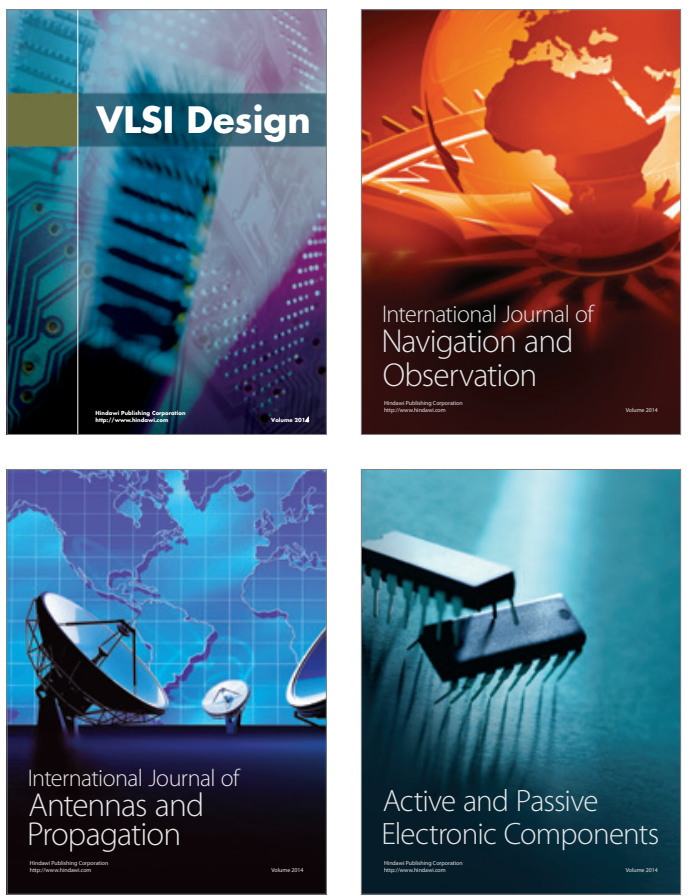
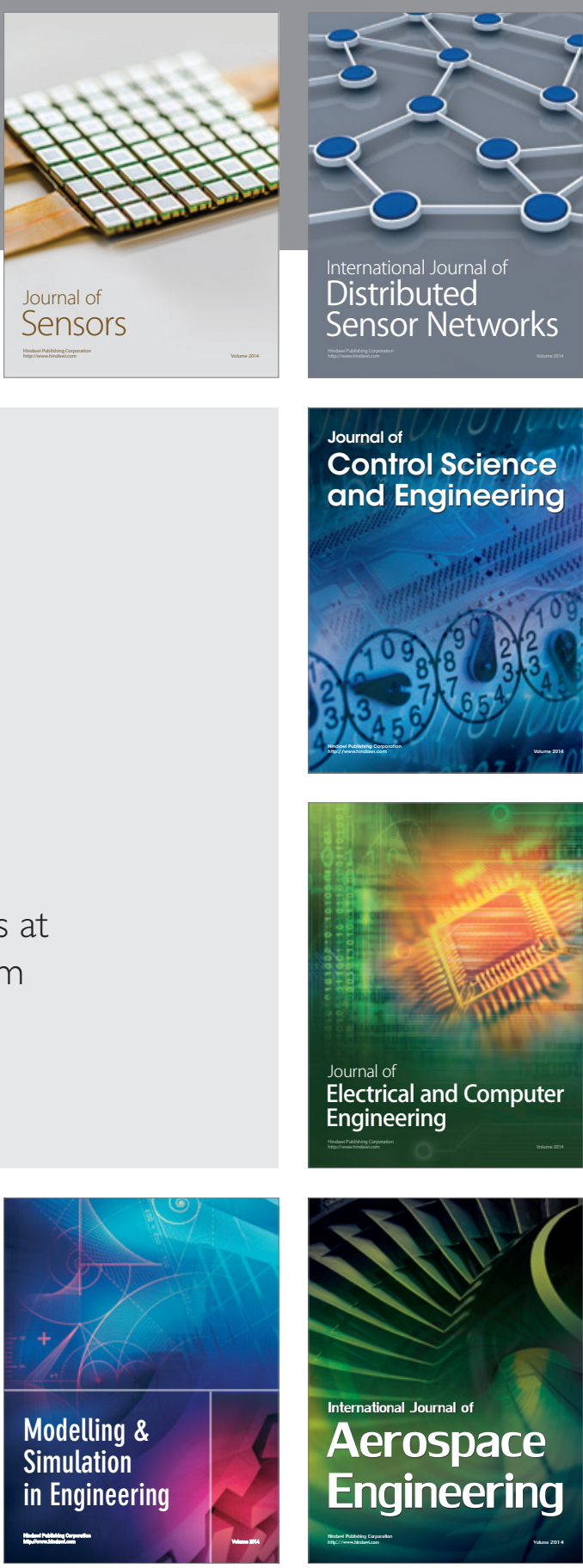

International Journal of

Distributed

Sensor Networks

Journal of

Control Science

and Engineering
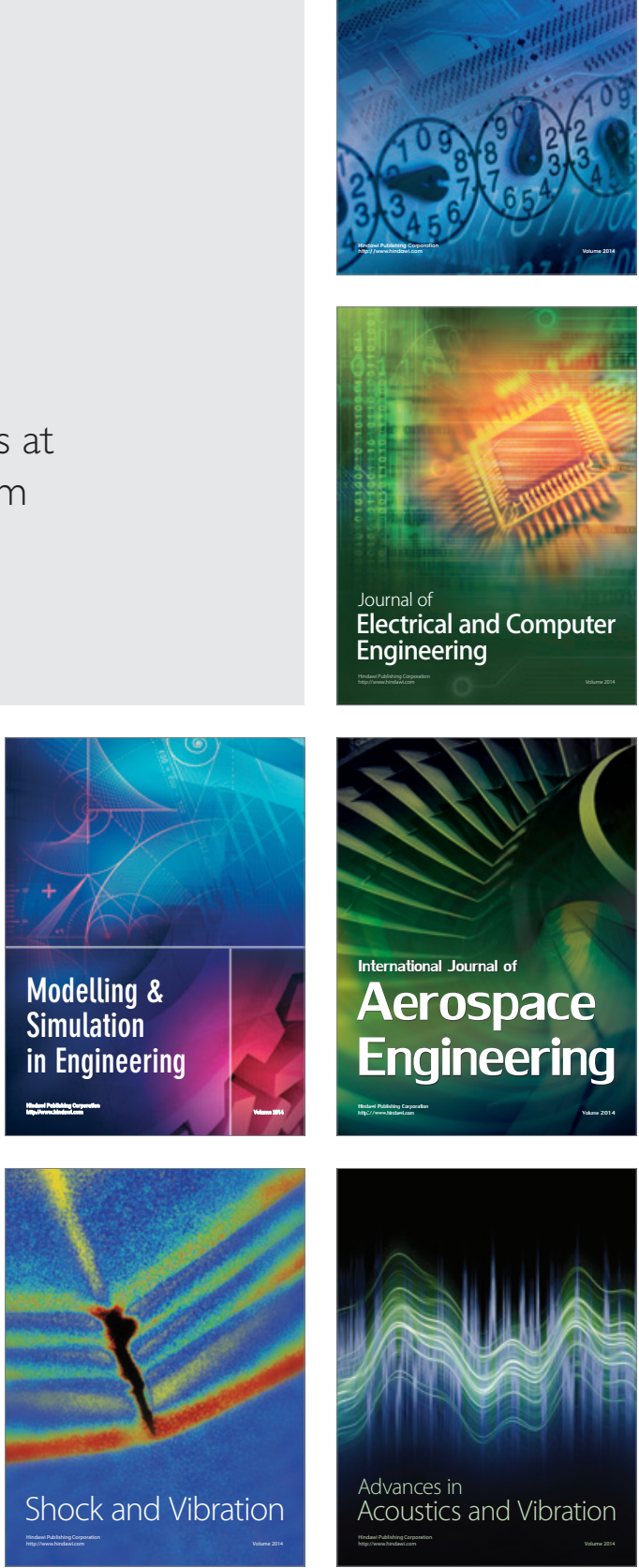